\title{
Bidirectionality in Bianistropic but Reciprocal Photonic Crystals and Its Usage in Active Photonics
}

\author{
Shu-Wei Chang, Member, IEEE
}

\begin{abstract}
We show that the bidirectionality of optical modes exists in general bianisotropic but reciprocal photonic crystals and related structures. Using this property, we obtain an alternative bidirectionality of modes with sources (gain) in active photonic crystals. In the former, we conclude that degeneracies of modes with complex anti-parallel wave vectors are always identical as long as the structure is reciprocal. With the latter, we setup an associated biorthogonality relation in the Rayleigh-Carson form for modes in active photonic crystals.
\end{abstract}

Index Terms-Bianisotropy, bidirectionality, biorthogonality, photonic crystal, reciprocity.

\section{INTRODUCTION}

$\mathbf{T}$ HE bidirectionality in ordered photonic structures is the symmetry between two groups of modes varying spatially along opposite directions. It serves as the foundation in many applications [1]-[9] such as mode expansions and excitations in waveguides. In homogeneous spaces and waveguides made of isotropic media, the bidirectionality is analytically reflected on the one-to-one correspondence between modes with wave vectors $\pm \mathbf{k}$ opposite in signs. The linkage between these modes, nevertheless, becomes nontrivial even in one-dimensional (1-D) bianisotropic waveguides and photonic crystals (PhCs) [10][18]. The complication lies in that in absence of symmetries, for example, the dissipation-free time-reversal symmetry $(\mathrm{T})$ for propagating modes [4], [17] (connected through the complex conjugation and proper sign changes of fields, analogous to the Kramers degeneracy in solids [19]-[21]) or inversion symmetry (P), there are often no direct ways to link modes with antiparallel wave vectors to each other in bianisotropic structures. Even if both categories of modes exist, their field distributions and polarization patterns may look unrelated, which complicates analyses.

In this study, we explore the bidirectionality and associated applications in general bianisotropic but reciprocal $\mathrm{PhCs}$ and related structures. First, we provide a a substitute approach to the bidirectionality in these structures in addition to the argument using fictitious analytic functions [12], [16] or a quite generic

Manuscript received July 7, 2013; revised October 8, 2013 and October 24, 2013; accepted October 30, 2013. Date of publication November 6, 2013; date of current version November 25, 2013. This work was supported in part by the research project of Research Center for Applied Sciences, Academia Sinica, Nankang, Taipei, Taiwan, and in part by the National Science Council, Taiwan, under Contract NSC102-2221-E-001-027.

The author is with the Research Center for Applied Sciences, Academia Sinica, Taipei 11529, Taiwan, and also with the Department of Photonics, National Chiao-Tung University, Hsinchu 30010, Taiwan (e-mail: swchang@ sinica.edu.tw)

Color versions of one or more of the figures in this paper are available online at http://ieeexplore.ieee.org.

Digital Object Identifier 10.1109/JLT.2013.2288982 method based on scattering matrices of antenna [17]. Second, utilizing the bidirectionality in reciprocal photonic structures, we present an alternative bidirectionality for modes with sources (gain) in active $\mathrm{PhCs}$, which originate from a generalized eigenvalue (GE) problem aimed at active photonic devices [22], [23]. Based on this new bidirectionality, we develop an associated mode biorthogonality relation in the Rayleigh-Carson form [24], [25]. This relation can be useful in the source/mode expansions and excitations in active PhCs.

Two points on the two aforementioned bidirectionalities in reciprocal (active) photonic structures require clarifications: (1) whether the existence of mode(s) at a wave vector $\mathbf{k ~ i m - ~}$ plies the presence of the counterpart(s) at $-\mathbf{k}$, and (2) if modes at $\pm \mathbf{k}$ both exist, are the two degeneracies identical (foundation of biorthogonality between modes at $\pm \mathbf{k}$ )? For the conventional bidirectionality, these two issues are addressed with a specialized form of matrix representations. Despite the bianisotropy, we can show that the degeneracies of modes with anti-parallel wave vectors, regardless of being propagating, evanescent, attenuated, or amplified, are identical as long as the structure is reciprocal. As to the alternative bidirectionality for active $\mathrm{PhCs}$, by maintaining the reciprocity of photonic structures, we transform the GE problem for active PhCs into an equivalent form in which the conventional bidirectionality is applicable. In this way, we assert that points (1) and (2) also hold positively for this new bidirectionality.

In the later part of this study, we will utilize plane-wave solutions in free spaces as an introduction of our approach to the bidirectionality (Section III) and then generalize it to the more complicated structures (Section IV). The procedure here also indicates how to maintain the bidirectionality in certain numerical calculations. In Section V, we present a 1-D chiral $\mathrm{PhC}$ with two alternating reciprocal chiral layers but lacking both $\mathrm{T}$ and $\mathrm{P}$ symmetries as an example of the bidirectionality. In Section VI, we utilize the conventional bidirectionality in reciprocal $\mathrm{PhCs}$ to obtain the alternative one and develop its associated biorthogonality relation.

\section{MaXwell's Equations IN SOURCe-Free But BIANISOTROPIC STRUCTURES}

The frequency-domain Maxwell's equations in source-free but bianisotopic structures can be expressed in the following matrix form as

$$
\begin{aligned}
\left(\begin{array}{cc}
\mathbf{0}_{3} & \nabla \times \\
\nabla \times & \mathbf{0}_{3}
\end{array}\right)\left(\begin{array}{c}
\mathbf{E}(\mathbf{r}) \\
\eta_{0} \mathbf{H}(\mathbf{r})
\end{array}\right)= & i\left(\frac{\omega}{c}\right)\left(\begin{array}{cc}
-\boldsymbol{\epsilon}_{\mathrm{r}}(\mathbf{r}, \omega) & -\boldsymbol{\xi}_{\mathrm{r}}(\mathbf{r}, \omega) \\
\boldsymbol{\zeta}_{\mathrm{r}}(\mathbf{r}, \omega) & \boldsymbol{\mu}_{\mathrm{r}}(\mathbf{r}, \omega)
\end{array}\right) \\
& \times\left(\begin{array}{c}
\mathbf{E}(\mathbf{r}) \\
\eta_{0} \mathbf{H}(\mathbf{r})
\end{array}\right)
\end{aligned}
$$


where $\mathbf{0}_{3}$ is the 3-by-3 null tensor; $\mathbf{E}(\mathbf{r})$ and $\mathbf{H}(\mathbf{r})$ are the electric and magnetic fields; $\omega$ is the frequency; $c$ is the speed of light in vacuum; $\eta_{0}$ is the intrinsic impedance; $\boldsymbol{\epsilon}_{\mathrm{r}}(\mathbf{r}, \omega)$ and $\boldsymbol{\mu}_{\mathrm{r}}(\mathbf{r}, \omega)$ are relative permittivity and permeability tensors, respectively; and $\boldsymbol{\zeta}_{\mathrm{r}}(\mathbf{r}, \omega)$ and $\boldsymbol{\xi}_{\mathrm{r}}(\mathbf{r}, \omega)$ are two dimensionless tensors in the constitutive relations. For reciprocal environments, the four tensors at the right-hand side (RHS) of (1) satisfy the following relations when they are represented in terms of real orthogonal unit vectors [26]:

$$
\begin{aligned}
& \boldsymbol{\epsilon}_{\mathrm{r}}(\mathbf{r}, \omega)=\boldsymbol{\epsilon}_{\mathrm{r}}^{\mathrm{T}}(\mathbf{r}, \omega), \quad \boldsymbol{\mu}_{\mathrm{r}}(\mathbf{r}, \omega)=\boldsymbol{\mu}_{\mathrm{r}}^{\mathrm{T}}(\mathbf{r}, \omega) \\
& \boldsymbol{\zeta}_{\mathrm{r}}(\mathbf{r}, \omega)=-\boldsymbol{\xi}_{\mathrm{r}}^{\mathrm{T}}(\mathbf{r}, \omega)
\end{aligned}
$$

where " $T$ " means the matrix transpose. With these constraints, the overall 6-by-6 tensor at RHS of (1) turns into a symmetric matrix.

The matrix partial differential equation in (1) and properties of various tensors in (2a) and (2b) serve as the foundation of our formalism in Sections III and IV. It should be emphasized that the resulted 6-by-6 symmetric tensor at RHS of (1) is essential to the bidirectionality in ordered bianisotropic photonic structures in which modes can be labeled with certain wave vectors $\mathbf{k}$.

\section{Bidirectionality IN FreE SpaceS}

We utilize plane-wave solutions in bianisotropic but reciprocal free spaces as an introduction to our approach. In homogeneous spaces, fields have a spatial form of $[\mathbf{E}(\mathbf{r}), \mathbf{H}(\mathbf{r})]=$ $[\mathcal{E}, \mathcal{H}] \exp (i \mathbf{k} \cdot \mathbf{r})$, where $\mathcal{E}$ and $\mathcal{H}$ are amplitudes of the respective fields, and $\mathbf{k}=k_{x} \hat{x}+k_{y} \hat{y}+k_{z} \hat{z}$ is a complex wave vector covering characteristics of propagating, evanescent, attenuated, and amplified waves. With this ansatz, Maxwell's equations in (1) become

$$
\begin{aligned}
\left(\begin{array}{cc}
\mathbf{0}_{3} & i \mathbf{C}[\mathbf{k}] \\
i \mathbf{C}[\mathbf{k}] & \mathbf{0}_{3}
\end{array}\right)\left(\begin{array}{c}
\mathcal{E} \\
\eta_{0} \mathcal{H}
\end{array}\right)= & i\left(\frac{\omega}{c}\right)\left(\begin{array}{cc}
-\boldsymbol{\epsilon}_{\mathrm{r}}(\omega) & -\boldsymbol{\xi}_{\mathrm{r}}(\omega) \\
-\boldsymbol{\xi}_{\mathrm{r}}^{\mathrm{T}}(\omega) & \boldsymbol{\mu}_{\mathrm{r}}(\omega)
\end{array}\right) \\
& \times\left(\begin{array}{c}
\mathcal{E} \\
\eta_{0} \mathcal{H}
\end{array}\right) \\
\mathbf{C}[\mathbf{k}]=-\mathbf{C}^{\mathrm{T}}[\mathbf{k}]=-\mathbf{C}[-\mathbf{k}]= & \left(\begin{array}{ccc}
0 & -k_{z} & k_{y} \\
k_{z} & 0 & -k_{x} \\
-k_{y} & k_{x} & 0
\end{array}\right)
\end{aligned}
$$

where $\mathbf{C}[\mathbf{k}]$ is a tensor due to actions of the curl operator $(\nabla \times)$ on the factor $\exp (i \mathbf{k} \cdot \mathbf{r})$ and is antisymmetric in the cartesian representation and odd in $\mathbf{k}$; and we have dropped the position (r) dependence on various tensors at RHS of (3a) due to homogeneous spaces. We further express (3a) more compactly with the composite vector $\mathbf{u}^{\mathrm{T}} \equiv\left[\mathcal{E}^{\mathrm{T}}, \eta_{0} \mathcal{H}^{\mathrm{T}}\right]$ as follows:

$$
\begin{aligned}
\mathbf{N}[\omega] \mathbf{u} & =-\mathbf{M}[\mathbf{k}] \mathbf{u} \\
\mathbf{N}[\omega] & =\left(\frac{\omega}{c}\right)\left(\begin{array}{cc}
\boldsymbol{\epsilon}_{\mathrm{r}}(\omega) & \boldsymbol{\xi}_{\mathrm{r}}(\omega) \\
\boldsymbol{\xi}_{\mathrm{r}}^{\mathrm{T}}(\omega) & -\boldsymbol{\mu}_{\mathrm{r}}(\omega)
\end{array}\right) \\
\mathbf{M}[\mathbf{k}] & =\left(\begin{array}{cc}
\mathbf{0}_{3} & \mathbf{C}[\mathbf{k}] \\
\mathbf{C}[\mathbf{k}] & \mathbf{0}_{3}
\end{array}\right) .
\end{aligned}
$$

Note that the 6-by-6 matrix $\mathbf{N}[\omega]$ is symmetric $\left(\mathbf{N}^{\mathrm{T}}[\omega]=\right.$ $\mathbf{N}[\omega])$ while $\mathbf{M}[\mathbf{k}]$ is antisymmetric $\left(\mathbf{M}^{\mathrm{T}}[\mathbf{k}]=-\mathbf{M}[\mathbf{k}]\right)$ and in particular, $\mathbf{M}^{\mathrm{T}}[\mathbf{k}]=\mathbf{M}[-\mathbf{k}]$.

Suppose that for a given frequency $\omega$ and possible wave vector $\mathbf{k}$, we have found all $d_{\mathbf{k}}(\omega)\left[d_{\mathbf{k}}(\omega) \geq 1\right]$ solutions $\mathbf{u}_{n}$ [ $n=1$ to $\left.d_{\mathbf{k}}(\omega)\right]$ to $(4 \mathrm{a})$, namely, $d_{\mathbf{k}}(\omega)$ is the degeneracy corresponding to the wave vector $\mathbf{k}$. These vectors $\mathbf{u}_{n}$, in fact, span the kernel (null space) of the matrix $\mathbf{N}[\omega]+\mathbf{M}[\mathbf{k}]$. From this point, the degeneracy $d_{\mathbf{k}}(\omega)$ is the dimension of this kernel (nullity). If we further utilize the matrix properties of $\mathbf{N}[\omega]$ and $\mathbf{M}[\mathbf{k}]$ and the fact that nullities (also ranks) of a matrix and its transpose are identical [27], we may rewrite $d_{\mathbf{k}}(\omega)$ as

$$
\begin{aligned}
d_{\mathbf{k}}(\omega) & =\operatorname{dim}[\operatorname{ker}\{\mathbf{N}[\omega]+\mathbf{M}[\mathbf{k}]\}] \\
& =\operatorname{dim}\left[\operatorname{ker}\left\{\mathbf{N}^{\mathrm{T}}[\omega]+\mathbf{M}^{\mathrm{T}}[\mathbf{k}]\right\}\right] \\
& =\operatorname{dim}[\operatorname{ker}\{\mathbf{N}[\omega]+\mathbf{M}[-\mathbf{k}]\}]
\end{aligned}
$$

where $\operatorname{ker}\{\mathbf{X}\}$ means the kernel of matrix $\mathbf{X} ; \operatorname{dim}[\mathbb{V}]$ is the dimension of vector space $\mathbb{V}$. The last line on (5) indicates that there exist exactly $d_{\mathbf{k}}(\omega)$ vectors $\mathbf{u}_{n}^{\prime}\left[n=1\right.$ to $\left.d_{\mathbf{k}}(\omega)\right]$ that span the kernel of $\mathbf{N}[\omega]+\mathbf{M}[-\mathbf{k}]$, and therefore these $d_{\mathbf{k}}(\omega)$ vectors $\mathbf{u}_{n}^{\prime}$ are just the plane-wave solutions at $-\mathbf{k}$. Thus, for the composite amplitude $\mathbf{u}^{\prime \mathrm{T}} \equiv\left[\mathcal{E}^{\prime \mathrm{T}}, \eta_{0} \mathcal{H}^{\prime \mathrm{T}}\right]$ satisfying the matrix equation at $-\mathbf{k}$ :

$$
\mathbf{N}[\omega] \mathbf{u}^{\prime}=-\mathbf{M}[-\mathbf{k}] \mathbf{u}^{\prime}
$$

the corresponding degeneracy $d_{-\mathbf{k}}(\omega)$ must be the same as the counterpart $d_{\mathbf{k}}(\omega)$ at $\mathbf{k}$, namely,

$$
d_{-\mathbf{k}}(\omega)=d_{\mathbf{k}}(\omega) .
$$

(7) indicates that in bianisotropic but reciprocal homogeneous spaces, mode degeneracies at wave vectors $\pm \mathbf{k}$ are identical to each other, and the bidirectionality in such spaces is proven. On the other hand, the proof says nothing about the explicit linkage between two solution groups of the composite amplitudes $\mathbf{u}(\mathcal{E}$ and $\mathcal{H})$ and $\mathbf{u}^{\prime}\left(\mathcal{E}^{\prime}\right.$ and $\left.\mathcal{H}^{\prime}\right)$ at $\pm \mathbf{k}$. In fact, their polarization patterns may bear no trivial connections.

\section{Bidirectionality In Photonic CRYstals AND RELATED STRUCTURES}

The key step toward the bidirectionality in reciprocal homogeneous spaces is the construction of two operation matrices: one is symmetric $(\mathbf{N}[\omega]$ in $(4 b))$, and the other, when evaluated at $-\mathbf{k}$, is identical to its transpose at $\mathbf{k}(\mathbf{M}[\mathbf{k}]$ in $(4 \mathrm{c}))$. We may follow the same scenarios for the bidirectionality in bianisotropic but reciprocal PhCs. Since these structures are inhomogeneous, we no longer have the simple case of free spaces. Rather, we need to construct the two matrices with a certain set of basis functions which expand the fields $\mathbf{E}(\mathbf{r})$ and $\mathbf{H}(\mathbf{r})$. The resulted operation matrices have sizes proportional to the number of basis functions included in the construction. If the symmetry and antisymmetry (as well as oddness in $\mathbf{k}$ ) of the two respective operation matrices are properly maintained as the number of basis functions increases, the bidirectionality of bianisotropic but reciprocal $\mathrm{PhCs}$ would follow naturally in the limit of infinitely many basis functions. 
There are many candidate sets of basis functions, but not each set can bring about desired matrix conditions. The failure in one basis set does not, however, mean that the bidirectionality is absent. As long as there exists one particular set that fulfills the requirement in its representations of the matrices, the bidirectionality in bianisotropic but reciprocal $\mathrm{PhCs}$ is granted. Representations in terms of other basis sets can be properly transformed into those of the very set that fulfills desired matrix conditions through some similarity transformations. Thus, the bidirectionality may be already embedded in the matrix equation regardless of the basis sets adopted, even though it is not always transparent from appearances of constructed matrices.

Let us consider a three-dimensional (3D) bianisotropic but reciprocal $\mathrm{PhC}$ of which the unit cell is characterized by three primitive vectors $\mathbf{R}_{m}$ ( $m=1$ to 3 ). The tensors $\boldsymbol{\epsilon}_{\mathrm{r}}(\mathbf{r}, \omega), \boldsymbol{\mu}_{\mathrm{r}}(\mathbf{r}, \omega)$, and $\boldsymbol{\xi}_{\mathrm{r}}(\mathbf{r}, \omega)=-\boldsymbol{\zeta}_{\mathrm{r}}^{\mathrm{T}}(\mathbf{r}, \omega)$ are periodic with respect to $\mathbf{R}_{m}$, namely,

$$
\begin{aligned}
& \boldsymbol{\epsilon}_{\mathrm{r}}\left(\mathbf{r}+\mathbf{R}_{m}, \omega\right)=\boldsymbol{\epsilon}_{\mathrm{r}}(\mathbf{r}, \omega), \quad \boldsymbol{\mu}_{\mathrm{r}}\left(\mathbf{r}+\mathbf{R}_{m}, \omega\right)=\boldsymbol{\mu}_{\mathrm{r}}(\mathbf{r}, \omega) \\
& \boldsymbol{\xi}_{\mathrm{r}}\left(\mathbf{r}+\mathbf{R}_{m}, \omega\right)=\boldsymbol{\xi}_{\mathrm{r}}(\mathbf{r}, \omega) .
\end{aligned}
$$

From the Bloch theorem, the fields $\mathbf{E}(\mathbf{r})$ and $\mathbf{H}(\mathbf{r})$ can be written in the Bloch form as

$$
\begin{aligned}
\mathbf{E}(\mathbf{r})=e^{i \mathbf{k} \cdot \mathbf{r}} \mathbf{e}(\mathbf{r}), & \mathbf{e}\left(\mathbf{r}+\mathbf{R}_{m}\right)=\mathbf{e}(\mathbf{r}) \\
\mathbf{H}(\mathbf{r})=e^{i \mathbf{k} \cdot \mathbf{r}} \mathbf{h}(\mathbf{r}), & \mathbf{h}\left(\mathbf{r}+\mathbf{R}_{m}\right)=\mathbf{h}(\mathbf{r})
\end{aligned}
$$

where $\mathbf{k}$ is a complex wave vector, which are not necessarily real and restricted to the first Brillouin zone (BZ) at this stage; and $\mathbf{e}(\mathbf{r})$ and $\mathbf{h}(\mathbf{r})$ are the Bloch periodic parts of $\mathbf{E}(\mathbf{r})$ and $\mathbf{H}(\mathbf{r})$, respectively. Corresponding to $\mathbf{R}_{m}$, the primitive reciprocal vectors $\mathbf{Q}_{n}$ ( $n=1$ to 3 ) in the reciprocal space are

$$
\mathbf{Q}_{n}=2 \pi \frac{\mathbf{R}_{u} \times \mathbf{R}_{v}}{\mathbf{R}_{1} \cdot\left(\mathbf{R}_{2} \times \mathbf{R}_{3}\right)}
$$

where $(n, u, v)$ are cyclic of $(1,2,3)$; and $\mathbf{R}_{m} \cdot \mathbf{Q}_{n}=2 \pi \delta_{m n}$.

Substituting (9a) and (9b) into (1), we obtain the matrix form of partial differential equations for $\mathbf{e}(\mathbf{r})$ and $\mathbf{h}(\mathbf{r})$ as follows:

$$
\begin{aligned}
& \left(\begin{array}{cc}
\mathbf{0}_{3} & \mathbf{C}[\mathbf{k}] \\
\mathbf{C}[\mathbf{k}] & \mathbf{0}_{3}
\end{array}\right)\left(\begin{array}{c}
\mathbf{e}(\mathbf{r}) \\
\eta_{0} \mathbf{h}(\mathbf{r})
\end{array}\right)=i\left(\begin{array}{cc}
\mathbf{0}_{3} & \nabla \times \\
\nabla \times & \mathbf{0}_{3}
\end{array}\right)\left(\begin{array}{c}
\mathbf{e}(\mathbf{r}) \\
\eta_{0} \mathbf{h}(\mathbf{r})
\end{array}\right) \\
& +\left(\frac{\omega}{c}\right)\left(\begin{array}{cc}
-\boldsymbol{\epsilon}_{\mathrm{r}}(\mathbf{r}, \omega) & -\boldsymbol{\xi}_{\mathrm{r}}(\mathbf{r}, \omega) \\
-\boldsymbol{\xi}_{\mathrm{r}}^{\mathrm{T}}(\mathbf{r}, \omega) & \boldsymbol{\mu}_{\mathrm{r}}(\mathbf{r}, \omega)
\end{array}\right) \\
& \times\left(\begin{array}{c}
\mathbf{e}(\mathbf{r}) \\
\eta_{0} \mathbf{h}(\mathbf{r})
\end{array}\right) .
\end{aligned}
$$

Analogous to the case of free spaces, we rewrite (11) with the composite amplitude $\mathbf{u}^{\mathrm{T}}(\mathbf{r}) \equiv\left[\mathbf{e}^{\mathrm{T}}(\mathbf{r}), \eta_{0} \mathbf{h}^{\mathrm{T}}(\mathbf{r})\right]$ as

$$
\begin{aligned}
\mathbf{F}[\mathbf{r}, \nabla, \omega] \mathbf{u}(\mathbf{r}) & =-\mathbf{M}[\mathbf{k}] \mathbf{u}(\mathbf{r}) \\
\mathbf{F}[\mathbf{r}, \nabla, \omega] & \equiv-i\left(\begin{array}{cc}
\mathbf{0}_{3} & \nabla \times \\
\nabla \times & \mathbf{0}_{3}
\end{array}\right)+\mathbf{N}[\mathbf{r}, \omega] \\
\mathbf{N}[\mathbf{r}, \omega] & =\left(\frac{\omega}{c}\right)\left(\begin{array}{cc}
\boldsymbol{\epsilon}_{\mathrm{r}}(\mathbf{r}, \omega) & \boldsymbol{\xi}_{\mathrm{r}}(\mathbf{r}, \omega) \\
\boldsymbol{\xi}_{\mathrm{r}}^{\mathrm{T}}(\mathbf{r}, \omega) & -\boldsymbol{\mu}_{\mathrm{r}}(\mathbf{r}, \omega)
\end{array}\right) .
\end{aligned}
$$

The amplitude $\mathbf{u}(\mathbf{r})$ and tensor $\mathbf{N}[\mathbf{r}, \omega]$ are spatially periodic, and additionally, $\mathbf{N}[\mathbf{r}, \omega]$ is symmetric $\left(\mathbf{N}[\mathbf{r}, \omega]=\mathbf{N}^{\mathrm{T}}[\mathbf{r}, \omega]\right)$.
Our goal is to find a basis set which makes the matrix representation of $\mathbf{F}[\mathbf{r}, \nabla, \omega]$ symmetric and that of $\mathbf{M}^{\mathrm{T}}[\mathbf{k}]$ identical to the counterpart of $\mathbf{M}[-\mathbf{k}]$.

A choice of basis sets is the collection of plane waves $\left\{\exp \left(i \mathbf{K}_{\mathbf{l}} \cdot \mathbf{r}\right)\right\}$ commonly adopted in plane-wave expansions of $\mathrm{PhC}$ modes, where $\mathbf{K}_{\mathbf{l}}$ is the reciprocal wave vector labeled by index $\mathbf{l}=\left(l_{1}, l_{2}, l_{3}\right),\left(l_{1}, l_{2}, l_{3} \in \mathbb{Z}\right)$ :

$$
\mathbf{K}_{\mathbf{l}}=l_{1} \mathbf{Q}_{1}+l_{2} \mathbf{Q}_{2}+l_{3} \mathbf{Q}_{3} .
$$

A quick observation reveals, however, that the basis $\exp \left(i \mathbf{K}_{1} \cdot \mathbf{r}\right)$ does not make the representation of $\mathbf{F}[\mathbf{r}, \nabla, \omega]$ symmetric. The failure arises partly from the action of matrix curl operator on $\exp \left(i \mathbf{K}_{\mathbf{l}} \cdot \mathbf{r}\right)$ at RHS of (12b):

$$
\begin{aligned}
-i\left(\begin{array}{cc}
\mathbf{0}_{3} & \nabla \times \\
\nabla \times & \mathbf{0}_{3}
\end{array}\right) & \rightarrow\left(\begin{array}{cc}
\mathbf{0}_{3} & \mathbf{C}\left[\mathbf{K}_{\mathbf{l}}\right] \\
\mathbf{C}\left[\mathbf{K}_{\mathbf{l}}\right] & \mathbf{0}_{3}
\end{array}\right) \\
& =\mathbf{M}\left[\mathbf{K}_{\mathbf{l}}\right]=-\mathbf{M}^{\mathrm{T}}\left[\mathbf{K}_{\mathbf{l}}\right]
\end{aligned}
$$

which appears antisymmetric in its diagonal blocks. In addition, the representation of $\mathbf{N}[\mathbf{r}, \omega]$ is not always symmetric in this basis set. Both factors potentially make the representation of $\mathbf{F}[\mathbf{r}, \nabla, \omega]$ asymmetric, and we need to check whether other basis sets can make both the representations of the matrix curl operator and $\mathbf{N}[\mathbf{r}, \omega]$ symmetric.

Rather than the plane-wave set, we adopt collections of cosines and sines as our basis set:

$$
\left\{\frac{\sqrt{2} \cos \left(\mathbf{K}_{\mathbf{l}} \cdot \mathbf{r}\right)}{\sqrt{1+\delta_{\mathrm{l}, 0}}}\right\}^{\prime},\left\{\sqrt{2} \sin \left(\mathbf{K}_{\mathbf{l}} \cdot \mathbf{r}\right)\right\}_{\mathbf{l} \neq \mathbf{0}}^{\prime}
$$

where the prime $\left({ }^{\prime}\right)$ means that only half the wave vectors $\mathbf{K}_{\mathbf{l}}$ in the reciprocal space is included because $\cos \left(\mathbf{K}_{1} \cdot \mathbf{r}\right)$ and $\cos \left(\mathbf{K}_{-\mathbf{l}} \cdot \mathbf{r}\right)\left[\sin \left(\mathbf{K}_{\mathbf{l}} \cdot \mathbf{r}\right)\right.$ and $\left.\sin \left(\mathbf{K}_{-\mathbf{l}} \cdot \mathbf{r}\right)\right]$ are linearly dependent; and the presence of Kronecker's delta $\delta_{1,0}$ for the basis function $\cos \left(\mathbf{K}_{\mathbf{0}} \cdot \mathbf{r}\right)=1$ is to make the spatial normalization of basis functions independent of index $\mathbf{l}$. We then expand the composite amplitude $\mathbf{u}(\mathbf{r})$ with cosine and sine functions as

$$
\mathbf{u}(\mathbf{r})=\sum_{1} \frac{\sqrt{2} \cos \left(\mathbf{K}_{1} \cdot \mathbf{r}\right)}{\sqrt{1+\delta_{1,0}}} \mathbf{U}_{1}^{(\mathrm{c})}+\sum_{\mathbf{l} \neq \mathbf{0}}^{\prime} \sqrt{2} \sin \left(\mathbf{K}_{\mathbf{l}} \cdot \mathbf{r}\right) \mathbf{U}_{1}^{(\mathrm{s})}
$$

where $\mathbf{U}_{1}^{(\mathrm{c})}$ and $\mathbf{U}_{1}^{(\mathrm{s})}$ are the expansion vectors for cosine and sine functions, respectively; and primes again indicate that only half of the wave vectors $\mathbf{K}_{\mathbf{l}}$ in the reciprocal space is included in the summation. For conveniences, we define a new amplitude $\mathbf{U}_{1}^{\mathrm{T}} \equiv\left[\mathbf{U}_{1}^{(\mathrm{c}) \mathrm{T}}, \mathbf{U}_{1}^{(\mathrm{s}) \mathrm{T}}\right]$ for the subspace of $\mathbf{l} \neq \mathbf{0}$ and demand $\mathbf{U}_{0} \equiv \mathbf{U}_{0}^{(\mathrm{c})}$. The representation $\mathcal{U}$ of the amplitude $\mathbf{u}(\mathbf{r})$ in terms of the basis set of cosine and sine functions is then defined as

$$
\mathcal{U}^{\mathrm{T}} \equiv\left[\ldots, \mathbf{U}_{\mathrm{l}_{1}}^{\mathrm{T}}, \mathbf{U}_{\mathbf{l}_{2}}^{\mathrm{T}}, \mathbf{U}_{\mathrm{l}_{3}}^{\mathrm{T}}, \ldots\right] .
$$

For the operation of an arbitrary 6-by-6 spatially periodic tensor operator $\mathbf{T}[\mathbf{r}, \nabla]$ on the amplitude $\mathbf{u}(\mathbf{r})$, we may similarly expand the resulted amplitude $\mathbf{v}(\mathbf{r}) \equiv \mathbf{T}[\mathbf{r}, \nabla] \mathbf{u}(\mathbf{r})$ and define 
its representation $\mathcal{V}$ as

$$
\begin{aligned}
\mathbf{v}(\mathbf{r}) & \equiv \mathbf{T}[\mathbf{r}, \nabla] \mathbf{u}(\mathbf{r}) \\
& =\sum_{\mathbf{l}} \frac{\sqrt{2} \cos \left(\mathbf{K}_{\mathbf{l}} \cdot \mathbf{r}\right)}{\sqrt{1+\delta_{\mathbf{l}, 0}}} \mathbf{V}_{\mathbf{l}}^{(\mathrm{c})}+\sum_{\mathbf{l} \neq \mathbf{0}}^{\prime} \sqrt{2} \sin \left(\mathbf{K}_{\mathbf{l}} \cdot \mathbf{r}\right) \mathbf{V}_{\mathbf{l}}^{(\mathrm{s})} \\
\mathbf{V}_{\mathbf{l}}^{\mathrm{T}} & =\left[\mathbf{V}_{\mathbf{l}}^{(\mathrm{c}) \mathrm{T}}, \mathbf{V}_{\mathbf{l}}^{(\mathrm{s}) \mathrm{T}}\right],(\mathbf{l} \neq \mathbf{0}), \mathbf{V}_{\mathbf{0}}=\mathbf{V}_{\mathbf{0}}^{(\mathrm{c})} \\
\boldsymbol{V}^{\mathrm{T}} & \equiv\left[\ldots, \mathbf{V}_{\mathbf{l}_{1}}^{\mathrm{T}}, \mathbf{V}_{\mathbf{l}_{2}}^{\mathrm{T}}, \mathbf{V}_{\mathbf{l}_{3}}^{\mathrm{T}}, \ldots\right] .
\end{aligned}
$$

The representation $\mathcal{V}$ can be generally expressed as $\mathcal{V}=\mathcal{T} \mathcal{U}$, where the matrix $\mathcal{T}$ is composed of infinitely many submatrices $\mathcal{T}_{1,1^{\prime}}$ which connect $\mathcal{V}_{1}$ to $\mathcal{U}_{1^{\prime}}$ :

$$
\mathcal{T}=\left(\begin{array}{ccccc}
\ddots & \text { column l } & \ldots & \text { column l }^{\prime} & \ldots \\
\operatorname{rowl} & \ddots & \ldots & \mathcal{T}_{1, l^{\prime}} & \ldots \\
\ldots & \ldots & \ddots & \ldots & \ldots \\
\operatorname{row~} \mathbf{l}^{\prime} & \mathcal{T}_{\mathbf{l}^{\prime}, 1} & \ldots & \ddots & \ldots \\
\ldots & \ldots & \ldots & \ldots & \ddots
\end{array}\right)
$$

The matrix $\mathcal{T}$ is taken as the representation of the tensor $\mathbf{T}[\mathbf{r}, \nabla]$ in terms of cosine and sine functions. By observing the relation between the submatrix $\mathcal{T}_{1^{\prime}, 1}^{\mathrm{T}}$ and $\mathcal{T}_{1,1^{\prime}}$, we can then determine whether the representation matrix $\mathcal{T}$ is symmetric, antisymmetric, or asymmetric.

We first consider the action of the tensor operator $\mathbf{F}[\mathbf{r}, \nabla, \omega]$ on $\mathbf{u}(\mathbf{r})$. From (12b), we need to find representations of the matrix curl operator and $\mathbf{N}[\mathbf{r}, \omega]$ which constitute $\mathbf{F}[\mathbf{r}, \nabla, \omega]$. Acting the matrix curl operator on $\mathbf{u}(\mathbf{r})$ and noting that the differential operator converts $\cos \left(\mathbf{K}_{\mathbf{l}} \cdot \mathbf{r}\right)$ into $-\sin \left(\mathbf{K}_{\mathbf{l}} \cdot \mathbf{r}\right)$ and $\sin \left(\mathbf{K}_{\mathbf{l}} \cdot \mathbf{r}\right)$ into $\cos \left(\mathbf{K}_{\mathbf{l}} \cdot \mathbf{r}\right)$, respectively, we obtain

$$
\begin{aligned}
\left(\begin{array}{cc}
\mathbf{0}_{3} & \nabla \times \\
\nabla \times & \mathbf{0}_{3}
\end{array}\right) \mathbf{u}(\mathbf{r})= & \sum_{\mathbf{l} \neq \mathbf{0}}^{\prime} \sqrt{2} \cos \left(\mathbf{K}_{\mathbf{l}} \cdot \mathbf{r}\right) \mathbf{M}\left[\mathbf{K}_{\mathbf{l}}\right] \mathbf{U}_{\mathbf{l}}^{(\mathrm{s})} \\
& +\sum_{\mathbf{l} \neq \mathbf{0}}^{\prime} \sqrt{2} \sin \left(\mathbf{K}_{\mathbf{l}} \cdot \mathbf{r}\right)(-1) \mathbf{M}\left[\mathbf{K}_{\mathbf{l}}\right] \mathbf{U}_{\mathbf{l}}^{(\mathrm{c})}
\end{aligned}
$$

Denote the representation for the matrix curl operator as $\mathcal{C}$. A close inspection of (20) reveals that its submatrix $\mathcal{C}_{1,1^{\prime}}$ is

$$
\begin{aligned}
& \mathcal{C}_{\mathbf{l}, \mathbf{l}^{\prime}}=\delta_{\mathbf{l}, \mathbf{1}^{\prime}}\left(\begin{array}{cc}
\mathbf{0}_{6} & \mathbf{M}\left[\mathbf{K}_{\mathbf{l}}\right] \\
-\mathbf{M}\left[\mathbf{K}_{\mathbf{l}}\right] & \mathbf{0}_{6}
\end{array}\right), \quad \mathbf{1}, \mathbf{l}^{\prime} \neq \mathbf{0} \\
& \mathcal{C}_{\mathbf{l}, \mathbf{0}}^{\mathrm{T}}=\mathcal{C}_{\mathbf{0}, \mathbf{l}}=\left(\begin{array}{ll}
\mathbf{0}_{6} & \mathbf{0}_{6}
\end{array}\right), \quad \mathbf{l} \neq \mathbf{0} \\
& \mathcal{C}_{\mathbf{0}, \mathbf{0}}=\mathbf{0}_{6}
\end{aligned}
$$

where $\mathbf{0}_{6}$ is the 6-by-6 null matrix. Due to the antisymmetry $\mathbf{M}\left[\mathbf{K}_{\mathbf{l}}\right]=-\mathbf{M}^{\mathrm{T}}\left[\mathbf{K}_{\mathbf{l}}\right]$, the transposed submatrix $\mathcal{C}_{\mathbf{l}^{\prime}, \mathbf{l}}^{\mathrm{T}}\left(\mathbf{l}, \mathbf{l}^{\prime} \neq \mathbf{0}\right)$ is identical to $\mathcal{C}_{1,1^{\prime}}$ :

$$
\begin{aligned}
\mathcal{C}_{\mathbf{l}^{\prime}, \mathbf{l}}^{\mathrm{T}} & =\delta_{\mathbf{l}^{\prime}, \mathbf{l}}\left(\begin{array}{cc}
\mathbf{0}_{6}^{\mathrm{T}} & -\mathbf{M}^{\mathrm{T}}\left[\mathbf{K}_{\mathbf{l}^{\prime}}\right] \\
\mathbf{M}^{\mathrm{T}}\left[\mathbf{K}_{\mathbf{l}^{\prime}}\right] & \mathbf{0}_{6}^{\mathrm{T}}
\end{array}\right) \\
& =\delta_{\mathbf{l}, \mathbf{l}^{\prime}}\left(\begin{array}{cc}
\mathbf{0}_{6} & \mathbf{M}\left[\mathbf{K}_{\mathbf{l}}\right] \\
-\mathbf{M}\left[\mathbf{K}_{\mathbf{l}}\right] & \mathbf{0}_{6}
\end{array}\right)=\mathcal{C}_{\mathbf{l}, \mathbf{1}^{\prime}}, \quad \mathbf{1}, \mathbf{l}^{\prime} \neq \mathbf{0} .
\end{aligned}
$$

In addition, submatrics $\mathcal{C}_{0,1}, \mathcal{C}_{1,0}(\mathbf{l} \neq \mathbf{0})$, and $\mathcal{C}_{0,0}$ in $(21)$ exhibit the same symmetry property. Therefore, the representation matrix $\mathcal{C}$ of the matrix curl operator is symmetric in terms of cosine and sine functions, namely,

$$
\mathcal{C}^{\mathrm{T}}=\mathcal{C}
$$

The property in (23) is a crucial step toward the symmetric matrix representation of the tensor operator $\mathbf{F}[\mathbf{r}, \nabla, \omega]$, in contrast to the construction in the plane-wave basis.

For the operator $\mathbf{N}[\mathbf{r}, \omega]$, we expand $\mathbf{N}[\mathbf{r}, \omega] \mathbf{u}(\mathbf{r})$ in terms of cosine and sine series and obtain its representation $\mathcal{N}[\omega]$. The detail is presented in appendix $\mathrm{A}$, and the corresponding submatrix $\mathcal{N}_{1,1^{\prime}}[\omega]$ indicates that $\mathcal{N}[\omega]$ is symmetric:

$$
\mathcal{N}_{\mathbf{l}^{\prime}, 1}^{\mathrm{T}}[\omega]=\mathcal{N}_{1,1^{\prime}}[\omega], \quad \mathcal{N}^{\mathrm{T}}[\omega]=\mathcal{N}[\omega]
$$

With symmetric matrix representations $\mathcal{C}$ and $\mathcal{N}[\omega]$, we then define a symmetric representation $\mathcal{F}[\omega]$ for the tensor operator $\mathbf{F}[\mathbf{r}, \nabla, \omega]$ in terms of cosine and sine functions as

$$
\begin{aligned}
\mathcal{F}[\omega] & =-i \mathcal{C}+\mathcal{N}[\omega], \quad \mathcal{F}^{\mathrm{T}}[\omega]=\mathcal{F}[\omega] \\
\mathcal{F}_{1,1^{\prime}}[\omega] & =-i \mathcal{C}_{1,1^{\prime}}+\mathcal{N}_{1,1^{\prime}}[\omega], \quad \mathcal{F}_{1^{\prime}, 1}^{\mathrm{T}}[\omega]=\mathcal{F}_{1,1^{\prime}}[\omega] .
\end{aligned}
$$

It remains to show that the representation $\mathcal{M}[\mathbf{k}]$ of the tensor operator $\mathbf{M}[\mathbf{k}]$ does have the required properties. Since the tensor $\mathbf{M}[\mathbf{k}]$ affects amplitudes $\mathbf{U}_{1}^{(\mathrm{c})}$ and $\mathbf{U}_{1}^{(\mathrm{s})}$ rather than cosine and sine functions, it can be directly shown that the corresponding submatrix $\mathcal{M}_{1,1^{\prime}}[\mathbf{k}]$ is

$$
\begin{array}{ll}
\mathcal{M}_{\mathbf{l}, \mathbf{l}^{\prime}}[\mathbf{k}] & =\delta_{\mathbf{l , \mathbf { l } ^ { \prime }}}\left(\begin{array}{cc}
\mathbf{M}[\mathbf{k}] & \mathbf{0}_{6} \\
\mathbf{0}_{6} & \mathbf{M}[\mathbf{k}]
\end{array}\right), \quad \mathbf{1}, \mathbf{l}^{\prime} \neq \mathbf{0} \\
\mathcal{M}_{\mathbf{l}, \mathbf{0}}^{\mathrm{T}}[\mathbf{k}] & =\mathcal{M}_{\mathbf{0}, \mathbf{1}}[\mathbf{k}]=\left(\begin{array}{ll}
\mathbf{0}_{6} & \mathbf{0}_{6}
\end{array}\right), \quad \mathbf{l} \neq \mathbf{0} \\
\mathcal{M}_{\mathbf{0}, \mathbf{0}}[\mathbf{k}] & =\mathbf{M}[\mathbf{k}] .
\end{array}
$$

With $\mathbf{M}^{\mathrm{T}}[\mathbf{k}]=\mathbf{M}[-\mathbf{k}]=-\mathbf{M}[\mathbf{k}]$, one sees that the submatrix $\mathcal{M}_{1,1^{\prime}}[\mathbf{k}]$ exhibits the following properties

$$
\mathcal{M}_{\mathbf{1}^{\prime}, 1}^{\mathrm{T}}[\mathbf{k}]=\mathcal{M}_{1,1^{\prime}}[-\mathbf{k}]=-\mathcal{M}_{\mathbf{l}, \mathbf{l}^{\prime}}[\mathbf{k}] \text {. }
$$

Therefore, we do have the desired property for the representation $\mathcal{M}[\mathbf{k}]$ :

$$
\mathcal{M}^{\mathrm{T}}[\mathbf{k}]=\mathcal{M}[-\mathbf{k}]=-\mathcal{M}[\mathbf{k}]
$$

With matrix representations in terms of cosine and sine functions, the matrix partial differential equation in (11) turns into a matrix form with an infinite size:

$$
\mathcal{F}[\omega] \mathcal{U}=-\mathcal{M}[\mathbf{k}] \mathcal{U} .
$$

In real computations, we can only include finitely many cosine and sine functions in the expansion. In other words, only a subset $\mathbf{L}$ of all possible indices $\mathbf{l}$ (excluding the redundancy from $\pm \mathbf{l})$ can be taken into account. Denote the matrices, $\mathcal{C}^{(\mathbf{L})}, \mathcal{N}^{(\mathbf{L})}[\omega], \mathcal{F}^{(\mathbf{L})}[\omega], \mathcal{M}^{(\mathbf{L})}[\mathbf{k}]$, and vector $\mathcal{U}^{(\mathbf{L})}$ as the counterparts of $\mathcal{C}, \mathcal{N}[\omega], \mathcal{F}[\omega], \mathcal{M}[\mathbf{k}]$, and $\mathcal{U}$, respectively, when only the basis functions in $\mathbf{L}$ are used. These smaller matrices exhibit the same symmetry properties and $\mathbf{k}$ dependence as their infinitely-dimensional counterparts do because 
their submatrices are identical, in particular,

$$
\begin{aligned}
\mathcal{F}^{(\mathbf{L}) \mathrm{T}}[\omega] & =\mathcal{F}^{(\mathbf{L})}[\omega] \\
\mathcal{M}^{(\mathbf{L}) \mathrm{T}}[\mathbf{k}] & =\mathcal{M}^{(\mathbf{L})}[-\mathbf{k}] .
\end{aligned}
$$

Suppose that at a give frequency $\omega$ and possible wave vector $\mathbf{k}^{(\mathbf{L})}$, we have found all $d_{\mathbf{k}^{(\mathbf{L})}}(\omega)$ solutions of $\mathcal{U}^{(\mathbf{L})}$ to the following matrix equation in the subset $\mathbf{L}$ :

$$
\mathcal{F}^{(\mathbf{L})}[\omega] \mathcal{U}^{(\mathbf{L})}=-\mathcal{M}^{(\mathbf{L})}\left[\mathbf{k}^{(\mathbf{L})}\right] \mathcal{U}^{(\mathbf{L})} .
$$

By the same token as that of biansitropic but reciprocal free spaces in Section III, the degeneracy $d_{\mathbf{k}^{(\mathbf{L})}}(\omega)$ is the nullity of $\mathcal{F}^{(\mathbf{L})}[\omega]+\mathcal{M}^{(\mathbf{L})}\left[\mathbf{k}^{(\mathbf{L})}\right]$ and is identical to that of $\mathcal{F}^{(\mathbf{L})}[\omega]+$ $\mathcal{M}^{(\mathbf{L})}\left[-\mathbf{k}^{(\mathbf{L})}\right]$ :

$$
\begin{aligned}
d_{\mathbf{k}^{(\mathbf{L})}}(\omega) & =\operatorname{dim}\left[\operatorname{ker}\left\{\mathcal{F}^{(\mathbf{L})}[\omega]+\mathcal{M}^{(\mathbf{L})}\left[\mathbf{k}^{(\mathbf{L})}\right]\right\}\right] \\
& =\operatorname{dim}\left[\operatorname{ker}\left\{\mathcal{F}^{(\mathbf{L}) \mathrm{T}}[\omega]+\mathcal{M}^{(\mathbf{L}) \mathrm{T}}\left[\mathbf{k}^{(\mathbf{L})}\right]\right\}\right] \\
& =\operatorname{dim}\left[\operatorname{ker}\left\{\mathcal{F}^{(\mathbf{L})}[\omega]+\mathcal{M}^{(\mathbf{L})}\left[-\mathbf{k}^{(\mathbf{L})}\right]\right\}\right] .
\end{aligned}
$$

On the other hand, the nullity of $\mathcal{F}^{(\mathbf{L})}[\omega]+\mathcal{M}^{(\mathbf{L})}\left[-\mathbf{k}^{(\mathbf{L})}\right]$ is just the degeneracy $d_{-\mathbf{k}^{(\mathbf{L})}}(\omega)$ of the solutions $\mathcal{U}^{(\mathbf{L})^{\prime}}$ to the matrix equation at $-\mathbf{k}^{(\mathbf{L})}$ :

$$
\mathcal{F}^{(\mathbf{L})}[\omega] \mathcal{U}^{\prime(\mathbf{L})}=-\mathcal{M}^{(\mathbf{L})}\left[-\mathbf{k}^{(\mathbf{L})}\right] \mathcal{U}^{\prime(\mathbf{L})} .
$$

Thus, the two degeneracies $d_{+\mathbf{k}^{(\mathbf{L})}}(\omega)$ corresponding to the subset $\mathbf{L}$ are identical, namely,

$$
d_{\mathbf{k}^{(\mathbf{L})}}(\omega)=d_{-\mathbf{k}^{(\mathbf{L})}}(\omega)
$$

and therefore, the bidirectionality of matrix representations is always valid for any finite subsets $\mathbf{L}$.

As the number of indices $\mathbf{l}$ in the subset $\mathbf{L}$ increases so that the expansion in terms of cosine and sine series becomes more complete, the wave vector $\mathbf{k}^{(\mathbf{L})}$ approaches the exact vector $\mathbf{k}$ while the degeneracies $d_{ \pm \mathbf{k}(\mathbf{L})}(\omega)$ turn into the true values $d_{ \pm \mathbf{k}}(\omega)$, where we have presumed that their limits in the infinitelydimensional expansion exist. Under such circumstances, the real-space composite amplitudes $\mathbf{u}(\mathbf{r})$ and $\mathbf{u}^{\prime}(\mathbf{r})$, which are solutions of the Bloch parts in 3D bianisotropic but reciprocal $\mathrm{PhCs}$ in (11), can be also accurately restored from $\mathcal{U}^{(\mathrm{L})}$ and $\mathcal{U}^{\prime(\mathrm{L})}$. Since the bidirectionality is rigorously maintained as the subset $\mathbf{L}$ becomes more complete, its presence in 3D bianisotropic but reciprocal PhCs is expected.

For related structures such as $(\mathrm{PhC})$ waveguides, the approach to the bidirectionality follows the same route with some adjustments of basis functions. For example, if the first primitive vector $\mathbf{R}_{1}$ vanishes (translationally invariant along $\hat{\mathbf{R}}_{1}$, where $\hat{\mathbf{R}}_{1}$ is the unit vector along the direction of $\mathbf{R}_{1}$ ), we only keep basis functions of which the corresponding indices $\mathbf{l}$ have their components $l_{1}=0$ so that Bloch parts have no position dependence along $\hat{\mathbf{R}}_{1}$. On the other hand, if structures are aperiodic along $\hat{\mathbf{R}}_{1}\left(\left|\mathbf{R}_{1}\right| \rightarrow \infty\right.$, super cells), we require $\mathbf{k} \cdot \hat{\mathbf{R}}_{1}=0$. The cosine and sine expansions in the corresponding dimension turn into continuous inverse Fourier cosine and sine transformations in the limit $\left|\mathbf{R}_{1}\right| \rightarrow \infty$. An ambiguity on $d_{ \pm \mathbf{k}}(\omega) \rightarrow \infty$ for unbounded modes in open structures may occur in this case, and it

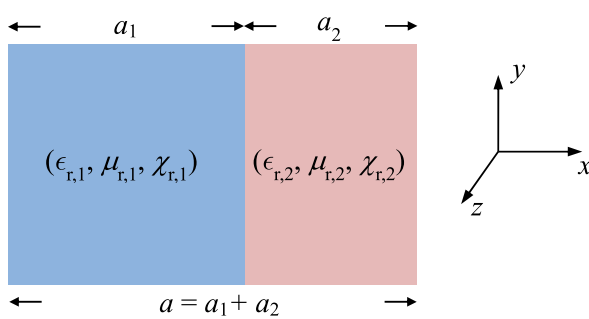

Fig. 1. The schematic diagram of a unit cell in the 1-D bilayer chiral $\mathrm{PhC}$. The unit cell is repeated in the $x$ direction.

becomes subtle to discuss whether two infinite degeneracies are identical. The situation arises because some modes with different wave vectors $\mathbf{k}$ at any finite $\mathbf{R}_{1}$ may merge into a continuum mode distribution as $\left|\mathbf{R}_{1}\right| \rightarrow \infty$. However, the bidirectionality at finite $\left|\mathbf{R}_{1}\right|$ indicates that two categories of modes around $\pm \mathbf{k}$ should evolve into respective continuums in an identical fashion as $\left|\mathbf{R}_{1}\right| \rightarrow \infty$. From this viewpoint, we expect that the bidirectionality should remain valid even if the reciprocal structure is open. Except for this subtlety, all other settings do not interfere with the required characteristics of operation matrices, and therefore the bidirectionality should be present in general bianistropic but reciprocal PhCs and related structures.

An accompanying remark is related to expansions based on the plane-wave set $\left\{\exp \left(i \mathbf{K}_{1} \cdot \mathbf{r}\right)\right\}$. Although this set does not directly lead to the bidirectionality in our formalism, for a given index $\mathbf{l} \neq \mathbf{0}$, both subsets $\left\{\exp \left(i \mathbf{K}_{\mathbf{l}} \cdot \mathbf{r}\right), \exp \left(-i \mathbf{K}_{\mathbf{l}} \cdot \mathbf{r}\right)\right\}$ and $\left\{\cos \left(\mathbf{K}_{1} \cdot \mathbf{r}\right), \sin \left(\mathbf{K}_{1} \cdot \mathbf{r}\right)\right\}$ expand the same subspace. Therefore, in numerical computations using plane-wave expansions, if two counter-propagating waves $\exp \left( \pm i \mathbf{K}_{\mathbf{l}} \cdot \mathbf{r}\right)(\mathbf{l} \neq \mathbf{0})$ are always included in pairs, the bidirectionality of photonic modes is automatically built in.

\section{EXAMPLE: Bilayer ChiRAL PHOTONIC CRYSTAL}

We utilize a 1-D PhC with alternating chiral slabs (biisotropic but reciprocal) as an example of the bidirectionality. As shown in Fig. 1, the unit cell of the bilayer PhC has a thickness $a$ and is repeated along the $x$ direction. Layers $w(w=1,2)$ in the unit cell has a thickness $a_{w}\left(a=a_{1}+a_{2}\right)$. In chiral structures, each tensor in the constitutive relation is diagonal and characterized by a scalar. For simplicity, these scalars are set frequency-independent. However, the relative permittivity $\epsilon_{\mathrm{r}, w}$ and permeability $\mu_{\mathrm{r}, w}$ in layer $w$ are set complex. In this way, the existing gain or loss in this PhC breaks the T symmetry even though under certain circumstances, the mode may still maintain a fixed magnitude as propagating through the whole structure. Also, in the constitutive relation, the scalar $\xi_{\mathrm{r}, w}$ in layer $w$ is replaced with the chiral parameter $\chi_{\mathrm{r}, w}\left(\xi_{\mathrm{r}, w}=i \chi_{\mathrm{r}, w}\right)$. We note that the spatial inversion $\mathrm{P}$ transforms the chiral structure to one with the opposite chirality, and therefore, the structure lacks the inversion symmetry. Hence, the bidirectionality in this $\mathrm{PhC}$ is not directly foreseeable using the arguments of T/P symmetries.

In the following calculations, we set the thicknesses of layers 1 and 2 as $a_{1}=2 a / 3$ and $a_{2}=a / 3$, respectively. Other parameters in layer 1 are set as follows: $\epsilon_{\mathrm{r}, 1}=(1.4-0.07 i)^{2}$; $\mu_{\mathrm{r}, 1}=(1+0.05 i)^{2} ;$ and $\chi_{\mathrm{r}, 1}=0.2$, and those in layer 2 are 

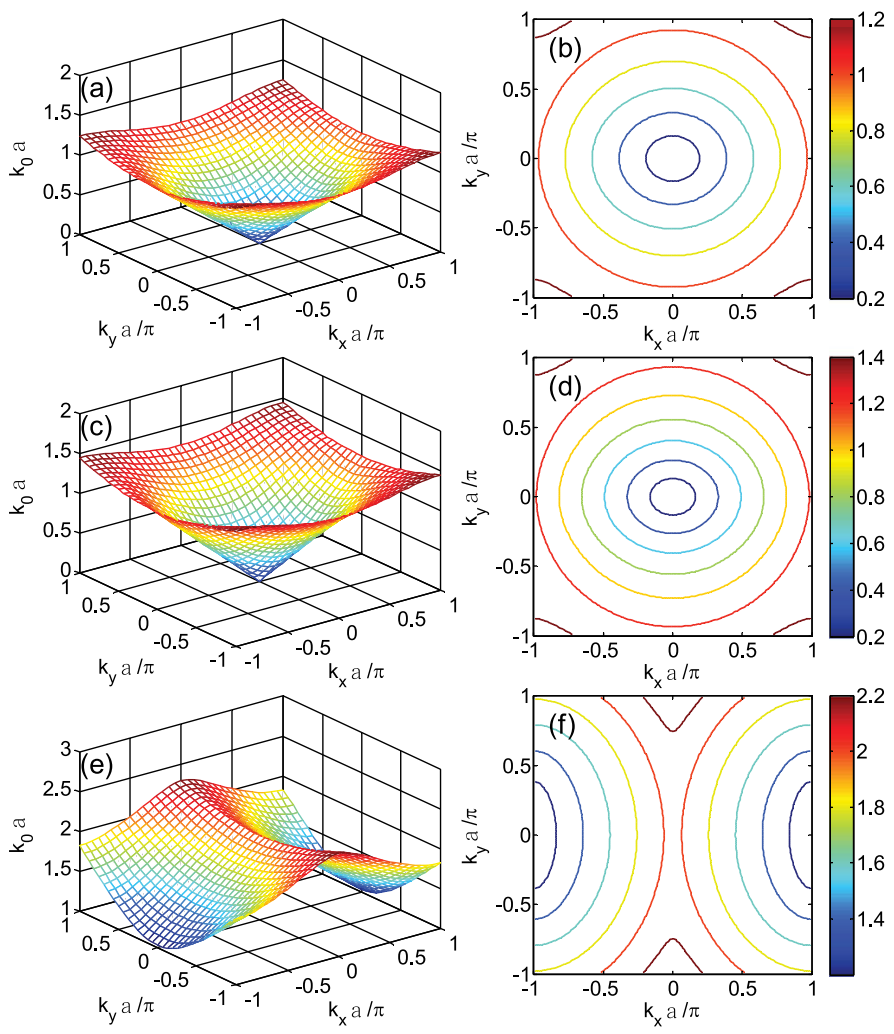

Fig. 2. Band diagrams (normalized frequency $k_{0} a$ ) and their iso-frequency contours along the plane of the normalized wave vectors $\left(k_{x} a / \pi-k_{y} a / \pi\right.$ plane) in the first BZ: (a) and (b), the band diagram and iso-frequency contour of the first band, respectively; (c) and (d), the counterparts from the second band; and (e) and (f), those of the third band. The three contours are all symmetric with respect to the origin in the $k_{x} a / \pi-k_{y} a / \pi$ plane, which is a sign of the bidirectionality of modes in this chiral and reciprocal $\mathrm{PhC}$.

$\epsilon_{\mathrm{r}, 2}=(2.8-0.14 i)^{2} ; \mu_{\mathrm{r}, 2}=(2+0.1 i)^{2} ;$ and $\chi_{\mathrm{r}, 2}=0.3$. The permittivity and permeability in each layer are chosen so that the electric gain exactly compensates the magnetic loss. In this way, the wave vectors of the propagation modes in this $\mathrm{PhC}$ remain real even if the system is time-irreversible. Here, we focus on the propagating modes but keep in mind that the bidirectionality still holds for evanescent ones. Also, since the slab structure is biisotropic and homogeneous in the $y-z$ plane, it is sufficient to consider the modes propagating in the $y$ direction and set $k_{z}$ to null.

The detail on the bandstructure calculations of this chiral $\mathrm{PhC}$ is presented in appendix B for interested readers. In Fig. 2, the band diagrams and corresponding iso-frequency contours of the first three bands in this $\mathrm{PhC}$ are depicted along the plane of normalized wave vectors $\left(k_{x} a / \pi-k_{y} a / \pi\right.$ plane $)$ in the first BZ. The frequency is also shown in a normalized form $k_{0} a$, where $k_{0}=\omega / c$ is the propagation constant in vacuum. The band diagrams can be further extended to $k_{y} \rightarrow \pm \infty$ because the chiral $\mathrm{PhC}$ is translationally invariant except for the $x$ direction. We note that these three bands themselves are not further degenerate. From Fig. 2(a), (c), and (e), although the dispersion relations of these three bands may look distinct, they all look symmetric as the normalized frequency at $\left(k_{x}, k_{y}\right)$ are mapped to that at $\left(-k_{x},-k_{y}\right)$ (inversion in the $\mathrm{k}$ space). In other words, whenever

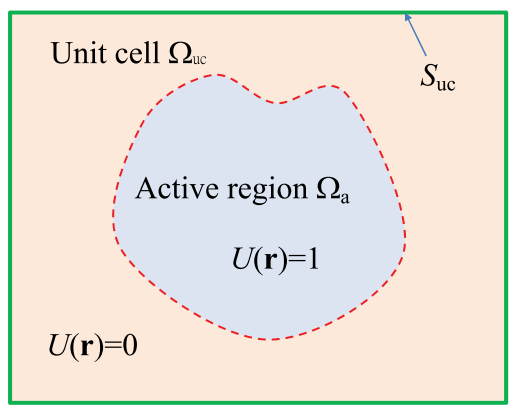

Fig. 3. The schematic diagram of a PhC unit cell. The function $U(\mathbf{r})$ is unity in $\Omega_{\mathrm{a}}$ but vanishes elsewhere.

there is a mode at wave vector $\mathbf{k}$, another at $-\mathrm{k}$ must be present. This property is more transparent from the corresponding isofrequency contours in Fig. 2(b), (d), and (f). These contours all have the inversion symmetry on the $k_{x} a / \pi-k_{y} a / \pi$ plane. These features are signs of the bidirectionality in this 1-D chiral and reciprocal $\mathrm{PhC}$, in which the $\mathrm{T}$ and $\mathrm{P}$ symmetries are absent.

\section{ALternative BidireCtionAlity AND ASSOCIATED BIORTHOGONALITY RELATION}

We further utilize the aforementioned bidirectionality in bianisotropic but reciprocal $\mathrm{PhCs}$ to demonstrate another type of bidirectionality and its associated biorthogonality relation in Rayleigh-Carson form for a GE problem developed for active photonic devices [22]. The construction of this GE problem is relevant to the mode expansion and source radiation in general reciprocal PhCs. In addition, the eigenvalue of this GE problem is directly related to the lasing threshold and coupling efficiency of active photonic modes [23] and may provide a guidance on how $\mathrm{PhC}$ structures should be designed for different applications and purposes.

Let us consider Maxwell's equations in bianisotropic but reciprocal $\mathrm{PhCs}$ with electric current sources $\mathbf{J}_{\mathrm{s}}(\mathbf{r})$ and magnetic counterparts $\mathbf{M}_{\mathrm{s}}(\mathbf{r})$ :

$$
\left\{\left(\begin{array}{cc}
\mathbf{0}_{3} & \nabla \times \\
\nabla \times & \mathbf{0}_{3}
\end{array}\right)+i \mathbf{N}[\mathbf{r}, \omega]\right\}\left(\begin{array}{c}
\mathbf{E}(\mathbf{r}) \\
\eta_{0} \mathbf{H}(\mathbf{r})
\end{array}\right)=\left(\begin{array}{c}
\eta_{0} \mathbf{J}_{\mathrm{s}}(\mathbf{r}) \\
-\mathbf{M}_{\mathbf{s}}(\mathbf{r})
\end{array}\right)
$$

For generic active photonic $\mathrm{PhCs}$, sources (gain) which generate radiation (amplification) are often confined in periodic active regions $\Omega_{\mathrm{a}}$ occupying a fraction of each unit cell $\Omega_{\mathrm{uc}}$, as indicated in Fig. 3. In particular, we demand sources $\mathbf{J}_{\mathrm{s}}(\mathbf{r})$ and $\mathbf{M}_{\mathrm{s}}(\mathbf{r})$ to have a specific form as follows:

$$
\begin{aligned}
\left(\begin{array}{c}
\eta_{0} \mathbf{J}_{\mathrm{s}}(\mathbf{r}) \\
-\mathbf{M}_{\mathrm{s}}(\mathbf{r})
\end{array}\right) & =-i\left(\frac{\omega}{c}\right) \Delta \gamma(\omega) U(\mathbf{r}) \mathbf{P}[\mathbf{r}, \omega]\left(\begin{array}{c}
\mathbf{E}(\mathbf{r}) \\
\eta_{0} \mathbf{H}(\mathbf{r})
\end{array}\right) \\
U(\mathbf{r}) & =\left\{\begin{array}{cc}
1, & \mathbf{r} \in \Omega_{\mathrm{a}} \\
0, & \text { otherwise }
\end{array}\right. \\
\mathbf{P}[\mathbf{r}, \omega] & =\left(\begin{array}{cc}
\boldsymbol{\kappa}_{\mathrm{r}}(\mathbf{r}, \omega) & \boldsymbol{\tau}_{\mathrm{r}}(\mathbf{r}, \omega) \\
\boldsymbol{\tau}_{\mathrm{r}}^{\mathrm{T}}(\mathbf{r}, \omega) & -\boldsymbol{\nu}_{\mathrm{r}}(\mathbf{r}, \omega)
\end{array}\right)
\end{aligned}
$$

where $\Delta \gamma(\omega)$ is a complex parameter; $U(\mathbf{r})$ is a periodic indicator function which is unity in $\Omega_{\mathrm{a}}$ but vanishes elsewhere; and 
$\mathbf{P}[\mathbf{r}, \omega]$ is a 6-by-6 periodic $\left(\mathbf{P}[\mathbf{r}, \omega]=\mathbf{P}\left[\mathbf{r}+\mathbf{R}_{m}, \omega\right]\right)$ and symmetric $\left(\mathbf{P}^{\mathrm{T}}[\mathbf{r}, \omega]=\mathbf{P}[\mathbf{r}, \omega]\right)$ tensor composed of 3-by-3 ones $\boldsymbol{\kappa}_{\mathrm{r}}(\mathbf{r}, \omega), \boldsymbol{\nu}_{\mathrm{r}}(\mathbf{r}, \omega)$, and $\boldsymbol{\tau}_{\mathrm{r}}(\mathbf{r}, \omega)$, of which the two formers are also symmetric:

$$
\begin{aligned}
& \boldsymbol{\kappa}_{\mathrm{r}}(\mathbf{r}, \omega)=\boldsymbol{\kappa}_{\mathrm{r}}\left(\mathbf{r}+\mathbf{R}_{m}, \omega\right)=\boldsymbol{\kappa}_{\mathrm{r}}^{\mathrm{T}}(\mathbf{r}, \omega) \\
& \boldsymbol{\nu}_{\mathrm{r}}(\mathbf{r}, \omega)=\boldsymbol{\nu}_{\mathrm{r}}\left(\mathbf{r}+\mathbf{R}_{m}, \omega\right)=\boldsymbol{\nu}_{\mathrm{r}}^{\mathrm{T}}(\mathbf{r}, \omega) \\
& \boldsymbol{\tau}_{\mathrm{r}}(\mathbf{r}, \omega)=\boldsymbol{\tau}_{\mathrm{r}}\left(\mathbf{r}+\mathbf{R}_{m}, \omega\right) .
\end{aligned}
$$

Substituting (36a) into RHS of (35), we obtain a GE problem in which parameter $\Delta \gamma(\omega)$ plays the role of eigenvalues. The interpretation of $\Delta \gamma(\omega)$ is more transparent if we group the tensor terms in $\mathbf{N}[\mathbf{r}, \omega]$ and $\mathbf{P}[\mathbf{r}, \omega]$ together. In this way, parameter $\Delta \gamma(\omega)$ is the required amount of tensor variations in $\Omega_{\mathrm{a}}$ that makes a certain $\mathrm{PhC}$ mode self oscillate at a real frequency $\omega$. The tensor variation is characterized by $\mathbf{P}[\mathbf{r}, \omega]$ $\left[\boldsymbol{\kappa}_{\mathrm{r}}(\mathbf{r}, \omega), \boldsymbol{\tau}_{\mathrm{r}}(\mathbf{r}, \omega)\right.$, and $\left.\boldsymbol{\nu}_{\mathrm{r}}(\mathbf{r}, \omega)\right]$. For example, if the PhC is originally passive, and we set $\boldsymbol{\kappa}_{\mathrm{r}}(\mathbf{r}, \omega)=\mathbf{I}_{3}$ (3-by-3 identity matrix) but $\boldsymbol{\tau}_{\mathrm{r}}(\mathbf{r}, \omega)=\boldsymbol{\nu}_{\mathrm{r}}(\mathbf{r}, \omega)=\mathbf{0}_{3}$, parameter $\Delta \gamma(\omega)$ is just the isotropic variation of the relative permittivity in $\Omega_{\mathrm{a}}$ that enables the oscillation of a certain mode at $\omega$, and its imaginary part $\operatorname{Im}[\Delta \gamma(\omega)]$ is related to the corresponding threshold gain [22], [23]. We note that the symmetric tensor $\mathbf{P}[\mathbf{r}, \omega]$ maintains the reciprocal form of the Maxwell's equation so that some of the results in Section IV are applicable here.

For modes in these active $\mathrm{PhCs}$, we express their fields and sources in the following Bloch forms as

$$
\begin{aligned}
& \left(\begin{array}{c}
\mathbf{E}(\mathbf{r}) \\
\eta_{0} \mathbf{H}(\mathbf{r})
\end{array}\right)=e^{i \mathbf{k} \cdot \mathbf{r}}\left(\begin{array}{c}
\boldsymbol{\psi}_{n \mathbf{k}}(\mathbf{r}, \omega) \\
\eta_{0} \boldsymbol{\varphi}_{n \mathbf{k}}(\mathbf{r}, \omega)
\end{array}\right) \equiv e^{i \mathbf{k} \cdot \mathbf{r}} \boldsymbol{\Psi}_{n \mathbf{k}}(\mathbf{r}, \omega) \\
& \left(\begin{array}{c}
\eta_{0} \mathbf{J}_{\mathrm{s}}(\mathbf{r}) \\
-\mathbf{M}_{\mathrm{s}}(\mathbf{r})
\end{array}\right)=e^{i \mathbf{k} \cdot \mathbf{r}}\left(\begin{array}{c}
\eta_{0} \boldsymbol{\varsigma}_{\mathrm{s}, n \mathbf{k}}(\mathbf{r}, \omega) \\
-\boldsymbol{\iota}_{\mathrm{s}, n \mathbf{k}}(\mathbf{r}, \omega)
\end{array}\right) \equiv e^{i \mathbf{k} \cdot \mathbf{r}} \boldsymbol{\Sigma}_{\mathrm{s}, n \mathbf{k}}(\mathbf{r}, \omega) \\
& \boldsymbol{\Sigma}_{\mathrm{s}, n \mathbf{k}}(\mathbf{r}, \omega)=-i\left(\frac{\omega}{c}\right) \Delta \gamma_{n \mathbf{k}}(\omega) U(\mathbf{r}) \mathbf{P}[\mathbf{r}, \omega] \mathbf{\Psi}_{n \mathbf{k}}(\mathbf{r}, \omega)
\end{aligned}
$$

where $n$ is the band label of Bloch modes; $\mathbf{k}$ is now restricted to real vectors in the first BZ since sources (gain) are present in the active region to sustain the field in each unit cell; $\boldsymbol{\psi}_{n \mathbf{k}}(\mathbf{r}, \omega), \boldsymbol{\varphi}_{n \mathbf{k}}(\mathbf{r}, \omega), \boldsymbol{\Psi}_{n \mathbf{k}}(\mathbf{r}, \omega)$ are Bloch periodic parts of $\mathbf{E}(\mathbf{r}), \mathbf{H}(\mathbf{r})$, and composite amplitude, respectively; and $\boldsymbol{\varsigma}_{\mathrm{s}, n \mathbf{k}}(\mathbf{r}, \omega), \boldsymbol{\iota}_{\mathrm{s}, n \mathbf{k}}(\mathbf{r}, \omega)$, and $\boldsymbol{\Sigma}_{\mathrm{s}, n \mathbf{k}}(\mathbf{r}, \omega)$ are the counterparts of $\mathbf{J}_{\mathrm{S}}(\mathbf{r}), \mathbf{M}_{\mathrm{S}}(\mathbf{r})$, and composite source. With (38), we convert the GE problem into the more compact form as

$$
\begin{aligned}
& \left\{i\left(\begin{array}{cc}
\mathbf{0}_{3} & \nabla \times \\
\nabla \times & \mathbf{0}_{3}
\end{array}\right)-\mathbf{N}[\mathbf{r}, \omega]-\mathbf{M}[\mathbf{k}]\right\} \boldsymbol{\Psi}_{n \mathbf{k}}(\mathbf{r}, \omega) \\
& =i \boldsymbol{\Sigma}_{\mathbf{s}, n \mathbf{k}}(\mathbf{r}, \omega) \\
& =\Delta \gamma_{n \mathbf{k}}(\omega) U(\mathbf{r})\left(\frac{\omega}{c}\right) \mathbf{P}[\mathbf{r}, \omega] \boldsymbol{\Psi}_{n \mathbf{k}}(\mathbf{r}, \omega)
\end{aligned}
$$

where the eigenvalue $\Delta \gamma_{n \mathbf{k}}(\omega)$ is now labeled with band index $n$ and wave vector $\mathbf{k}$. Our investigations on the biorthogonality then turn into the search of connections between (non)degenerate sets of eigenvalues $\Delta \gamma_{n \mathbf{k}}(\omega)$ and $\Delta \gamma_{n-\mathbf{k}}(\omega)$ at $\pm \mathbf{k}$.

Suppose that at a real wave vector $\mathbf{k}$ in the first BZ, we have exhausted all possible modes in a certain (non)degenerate set $\mathbf{n} \equiv\left\{n_{j}\right\}$, of which the degeneracy and representative eigenvalue are $D_{\mathrm{nk}}(\omega)$ and $\Delta \gamma_{\mathrm{nk}}(\omega)$, respectively, namely, all eigenvalues $\Delta \gamma_{n_{j} \mathbf{k}}(\omega)\left[j=1\right.$ to $\left.D_{\mathbf{n k}}(\omega)\right]$ of the modes in set $\mathbf{n}$ are identical to $\Delta \gamma_{\mathbf{n k}}(\omega)$. We may cast the GE problem in (39) into the form of matrix differential equations in (12a) by defining two tensor operators $\mathbf{N}^{\prime}[\mathbf{r}, \omega]$ and $\mathbf{F}^{\prime}[\mathbf{r}, \nabla, \omega]$ as

$$
\begin{aligned}
\mathbf{N}^{\prime}[\mathbf{r}, \omega] & =\mathbf{N}[\mathbf{r}, \omega]+\Delta \gamma_{\mathbf{n k}}(\omega) U(\mathbf{r})\left(\frac{\omega}{c}\right) \mathbf{P}[\mathbf{r}, \omega] \\
\mathbf{F}^{\prime}[\mathbf{r}, \nabla, \omega] & =-i\left(\begin{array}{cc}
\mathbf{0}_{3} & \nabla \times \\
\nabla \times & \mathbf{0}_{3}
\end{array}\right)+\mathbf{N}^{\prime}[\mathbf{r}, \omega] .
\end{aligned}
$$

Similar to $\mathbf{N}[\mathbf{r}, \omega]$, the tensor $\mathbf{N}^{\prime}[\mathbf{r}, \omega]$ is also symmetric $\left(\mathbf{N}^{\prime T}[\mathbf{r}, \omega]=\mathbf{N}^{\prime}[\mathbf{r}, \omega]\right)$ and periodic. With these new operators, (39) is rewritten as

$$
\mathbf{F}^{\prime}[\mathbf{r}, \nabla, \omega] \boldsymbol{\Psi}_{n \mathbf{k}}(\mathbf{r}, \omega)=-\mathbf{M}[\mathbf{k}] \boldsymbol{\Psi}_{n \mathbf{k}}(\mathbf{r}, \omega)
$$

Since all of the $D_{\mathbf{n k}}(\omega)$ solutions in set $\mathbf{n}$ have been identified, the enumeration of this/these (non)degenerate mode(s) must be reflected on the degeneracy $d_{\mathrm{k}}^{\prime}(\omega)$ corresponding to the matrix differential equation in (41) (nullity of the operator $\left.\mathbf{F}^{\prime}[\mathbf{r}, \nabla, \omega]+\mathbf{M}[\mathbf{k}]\right)$, namely,

$$
d_{\mathrm{k}}^{\prime}(\omega)=D_{\mathrm{nk}}(\omega)
$$

On the other hand, the bidirectionality of $\mathrm{PhC}$ modes in Section IV indicates that the counterpart $d_{-\mathbf{k}}^{\prime}(\omega)$ at $-\mathbf{k}$ is identical to $d_{\mathbf{k}}^{\prime}(\omega)$, and hence there are exactly $D_{\mathbf{n k}}(\omega)$ solutions $\boldsymbol{\Psi}_{n-\mathbf{k}}(\mathbf{r}, \omega)$ for the matrix differential equation at $-\mathbf{k}$ :

$$
\mathbf{F}^{\prime}[\mathbf{r}, \nabla, \omega] \boldsymbol{\Psi}_{n-\mathbf{k}}(\mathbf{r}, \omega)=-\mathbf{M}[-\mathbf{k}] \boldsymbol{\Psi}_{n-\mathbf{k}}(\mathbf{r}, \omega)
$$

Equivalently, $\boldsymbol{\Psi}_{n-\mathbf{k}}(\mathbf{r}, \omega)$ is an eigenvector of the GE problem with, however, an eigenvalue $\Delta \gamma_{\mathbf{n k}}(\omega)$ at $-\mathbf{k}$ :

$$
\begin{aligned}
& \left\{i\left(\begin{array}{cc}
\mathbf{0}_{3} & \nabla \times \\
\nabla \times & \mathbf{0}_{3}
\end{array}\right)-\mathbf{N}[\mathbf{r}, \omega]-\mathbf{M}[-\mathbf{k}]\right\} \boldsymbol{\Psi}_{n-\mathbf{k}}(\mathbf{r}, \omega) \\
& \quad=\Delta \gamma_{\mathbf{n k}}(\omega) U(\mathbf{r})\left(\frac{\omega}{c}\right) \mathbf{P}[\mathbf{r}, \omega] \Psi_{n-\mathbf{k}}(\mathbf{r}, \omega) .
\end{aligned}
$$

In other words, there are $D_{\mathrm{nk}}(\omega)$ eigenvectors $\boldsymbol{\Psi}_{n-\mathbf{k}}(\mathbf{r}, \omega)$ with their eigenvalues $\Delta \gamma_{n-\mathbf{k}}(\omega)=\Delta \gamma_{\mathbf{n k}}(\omega)$ at $-\mathbf{k}$. Except for these $D_{\mathrm{nk}}(\omega)$ eigenvectors, no additional ones at $-\mathbf{k}$ have eigenvalues $\Delta \gamma_{\mathbf{n k}}(\omega)$. Otherwise, the bidirectionality, when applied to these $\mathrm{PhC}$ modes at $-\mathbf{k}$, would imply that the number of eigenvectors $\boldsymbol{\Psi}_{n \mathbf{k}}(\mathbf{r}, \omega)$ with an eigenvalue of $\Delta \gamma_{\mathbf{n k}}(\omega)$ at k exceeds $D_{\mathrm{nk}}(\omega)$, which contradicts the presumption that all mode(s) in set $\mathbf{n}$ have been enumerated.

From the reasoning above, we may also denote the set of these $D_{\mathbf{n k}}(\omega)$ modes $\Psi_{n-\mathbf{k}}(\mathbf{r}, \omega)$ at $-\mathbf{k}$ as $\mathbf{n}$ because they are closely connected to their counterparts at $\mathbf{k}$ through the bidirectionality. In this way, the degeneracy $D_{\mathbf{n}-\mathbf{k}}(\omega)$ and representative eigenvalue $\Delta \gamma_{\mathbf{n}-\mathbf{k}}(\omega)$ of this (non)degenerate set $\mathbf{n}$ at $-\mathbf{k}$ are 
identical to their counterparts at $\mathbf{k}$, respectively:

$$
\begin{aligned}
D_{\mathbf{n}-\mathbf{k}}(\omega) & =D_{\mathbf{n k}}(\omega) \\
\Delta \gamma_{\mathbf{n}-\mathbf{k}}(\omega) & =\Delta \gamma_{\mathbf{n k}}(\omega) .
\end{aligned}
$$

(45a) and (45b) is the bidirectionality of the GE problem in active PhCs. As long as the PhCs and tensor variations in active regions are both reciprocal, the eigenvalues $\Delta \gamma_{n \mathbf{k}}(\omega)$ at $\mathbf{k}$ would all repeat at $-\mathrm{k}$ and vice versa. The degeneracies of these repeated eigenvalues at $\pm \mathbf{k}$ must be identical. Furthermore, from (45b), these counter-propagating modes have the same threshold (gain) regardless of their potentially distinct field (polarization) patterns and geometries of the active region.

Since the new bidirectionality guarantees that the related (non)degenerate sets at $\pm \mathbf{k}$ have the same degeneracies (degrees of freedom), we may safely develop a biorthogonality relation for eigenvectors from two parts. Following the derivation of the reciprocity theorem, we apply similar procedures to various Bloch periodic parts with mode labels $(n, \mathbf{k})$ and $\left(n^{\prime}, \mathbf{k}^{\prime}\right)$ as follows [22]:

$$
\begin{aligned}
& \oint_{S_{\mathrm{uc}}} \mathrm{d} \mathbf{a} \cdot[ \boldsymbol{\psi}_{n \mathbf{k}}(\mathbf{r}, \omega) \times \boldsymbol{\varphi}_{n^{\prime} \mathbf{k}^{\prime}}(\mathbf{r}, \omega) \\
&-\left.\boldsymbol{\psi}_{n^{\prime} \mathbf{k}^{\prime}}(\mathbf{r}, \omega) \times \boldsymbol{\varphi}_{n \mathbf{k}}(\mathbf{r}, \omega)\right] \\
&=-\int_{\Omega_{\mathrm{uc}}} \mathrm{d} \mathbf{r} i\left(\mathbf{k}+\mathbf{k}^{\prime}\right) \cdot\left[\boldsymbol{\psi}_{n \mathbf{k}}(\mathbf{r}, \omega) \times \boldsymbol{\varphi}_{n^{\prime} \mathbf{k}^{\prime}}(\mathbf{r}, \omega)\right. \\
&\left.-\boldsymbol{\psi}_{n^{\prime} \mathbf{k}^{\prime}}(\mathbf{r}, \omega) \times \boldsymbol{\varphi}_{n \mathbf{k}}(\mathbf{r}, \omega)\right] \\
&-\int_{\Omega_{\mathrm{uc}}} \mathrm{d} \mathbf{r}\left\{\left[\boldsymbol{\psi}_{n \mathbf{k}}(\mathbf{r}, \omega) \cdot \boldsymbol{\varsigma}_{\mathrm{s}, n^{\prime} \mathbf{k}^{\prime}}(\mathbf{r}, \omega)\right.\right. \\
&\left.-\boldsymbol{\varphi}_{n \mathbf{k}}(\mathbf{r}, \omega) \cdot \boldsymbol{\iota}_{\mathrm{s}, n^{\prime} \mathbf{k}^{\prime}}(\mathbf{r}, \omega)\right] \\
&-\left[\boldsymbol{\psi}_{n^{\prime} \mathbf{k}^{\prime}}(\mathbf{r}, \omega) \cdot \boldsymbol{\varsigma}_{\mathrm{s}, n \mathbf{k}}(\mathbf{r}, \omega)\right. \\
&\left.\left.-\boldsymbol{\varphi}_{n^{\prime} \mathbf{k}^{\prime}}(\mathbf{r}, \omega) \cdot \boldsymbol{\iota}_{\mathrm{s}, n \mathbf{k}}(\mathbf{r}, \omega)\right]\right\}
\end{aligned}
$$

where $S_{\mathrm{uc}}$ is the surface of the unit cell. The surface integral at the left-hand side of (46) is always zero because the integrand repeats at any opposite sides of the unit cell while the corresponding surface normals are antiparallel. If we further choose $\mathbf{k}^{\prime}=-\mathbf{k}$, the first volume integral at RHS of (46) also vanishes. The remaining volume integral, aside of a factor of $\eta_{0}$, can be compactly expressed in terms of the Bloch periodic parts of composite amplitudes $\boldsymbol{\Psi}_{n \mathbf{k}}(\mathbf{r}, \omega)$ and $\boldsymbol{\Psi}_{n^{\prime}-\mathbf{k}}(\mathbf{r}, \omega)$ as well as sources $\boldsymbol{\Sigma}_{\mathrm{s}, n \mathbf{k}}(\mathbf{r}, \omega)$ and $\boldsymbol{\Sigma}_{\mathrm{s}, n^{\prime}-\mathbf{k}}(\mathbf{r}, \omega)$. The consequence is a reciprocity relation in the Rayleigh-Carson form:

$$
\begin{aligned}
0=\int_{\Omega_{\mathrm{uc}}} \mathrm{d} \mathbf{r} & {\left[\boldsymbol{\Psi}_{n \mathbf{k}}^{\mathrm{T}}(\mathbf{r}, \omega) \boldsymbol{\Sigma}_{\mathrm{s}, n^{\prime}-\mathbf{k}}(\mathbf{r}, \omega)\right.} \\
& \left.-\boldsymbol{\Psi}_{n^{\prime}-\mathbf{k}}^{\mathrm{T}}(\mathbf{r}, \omega) \boldsymbol{\Sigma}_{\mathrm{s}, n \mathbf{k}}(\mathbf{r}, \omega)\right] .
\end{aligned}
$$

Further utilizing the form of $\boldsymbol{\Sigma}_{\mathrm{s}, n \mathbf{k}}(\mathbf{r}, \omega)\left[\boldsymbol{\Sigma}_{\mathrm{s}, n^{\prime}-\mathbf{k}}(\mathbf{r}, \omega)\right]$ in (38) and noting that $\mathbf{P}[\mathbf{r}, \omega]$ is symmetric, we obtain the following integral identity:

$$
\begin{aligned}
0= & -i\left(\frac{\omega}{c}\right)\left[\Delta \gamma_{n^{\prime}-\mathbf{k}}(\omega)-\Delta \gamma_{n \mathbf{k}}(\omega)\right] \\
& \times \int_{\Omega_{\mathrm{a}}} \mathrm{d} \mathbf{r} \boldsymbol{\Psi}_{n \mathbf{k}}^{\mathrm{T}}(\mathbf{r}, \omega) \mathbf{P}[\mathbf{r}, \omega] \Psi_{n^{\prime}-\mathbf{k}}(\mathbf{r}, \omega)
\end{aligned}
$$

where the volume integral on the second line is now carried out in $\Omega_{\mathrm{a}}$ within one unit cell due to the indicator function $U(\mathbf{r})$. From (48), if $\Delta \gamma_{n^{\prime}-\mathbf{k}}(\omega) \neq \Delta \gamma_{n \mathbf{k}}(\omega)$, the volume integral in $\Omega_{\mathrm{a}}$ has to vanish. On the other hand, if $\Delta \gamma_{n^{\prime}-\mathbf{k}}(\omega)=\Delta \gamma_{n \mathbf{k}}(\omega)$, which must occur for some band indices $n$ and $n^{\prime}$ belonging to a certain (non)degenerate set $\mathbf{n}$ due to the bidirectionality of the GE problem in (45b), we can still use the volume integral as a device to biorthogonalize these modes at $\pm \mathbf{k}$. The biorthogonalization can always proceed unambiguously because the numbers of modes (degrees of freedom) in a (non)degenerate set $\mathbf{n}$ at $\pm \mathbf{k}$ are granted to be identical. In other words, after the proper biorthogonalization, we can demand

$$
\int_{\Omega_{\mathrm{a}}} \mathrm{d} \mathbf{r} \Psi_{n \mathbf{k}}^{\mathrm{T}}(\mathbf{r}, \omega) \mathbf{P}[\mathbf{r}, \omega] \Psi_{n^{\prime}-\mathbf{k}}(\mathbf{r}, \omega)=\delta_{n n^{\prime}} \Lambda_{n \mathbf{k}}(\omega)
$$

where $\delta_{n n^{\prime}}$ is Kronecker's delta; and $\Lambda_{n \mathbf{k}}(\omega)$ is a normalization constant. (49) is the biorthogonality relation in the RayleighCarson form for modes in bianisotopic but reciprocal PhCs with sources (gain) in the active region.

\section{CONCLUSION}

In conclusions, we have demonstrated that the bidirectionality always exists in bianisotropic but reciprocal homogeneous spaces, PhCs, and related structures. Our approach is based on the construction of one symmetric operation matrix and another one, which, when evaluated at $-\mathbf{k}$, is identical to its transpose at $\mathbf{k}$ for wave equations of photonic modes. We also utilize this bidirectionality in reciprocal $\mathrm{PhCs}$ to show an alternative one for a GE problem developed for modes in PhCs with sources (gain). This new bidirectionality consolidates the usage of an associated biorthogonality relation in the Rayleigh-Carson form.

\section{APPENDIX A MATRIX REPRESENTATION $\mathcal{N}[\omega]$}

We denote the outcome of $\mathbf{N}[\mathbf{r}, \omega] \mathbf{u}(\mathbf{r})$ as $\mathbf{g}(\mathbf{r})$ :

$$
\begin{aligned}
\mathbf{g}(\mathbf{r})= & \sum_{\mathbf{l}} \frac{\sqrt{2} \cos \left(\mathbf{K}_{\mathbf{l}} \cdot \mathbf{r}\right)}{\sqrt{1+\delta_{\mathbf{l}, 0}}} \mathbf{N}[\mathbf{r}, \omega] \mathbf{U}_{\mathbf{l}}^{(\mathrm{c})} \\
& +\sum_{\mathbf{l} \neq \mathbf{0}}{ }^{\prime} \sqrt{2} \sin \left(\mathbf{K}_{\mathbf{l}} \cdot \mathbf{r}\right) \mathbf{N}[\mathbf{r}, \omega] \mathbf{U}_{1}^{(\mathrm{s})} \\
\equiv & \sum_{\mathbf{l}}, \frac{\sqrt{2} \cos \left(\mathbf{K}_{\mathbf{l}} \cdot \mathbf{r}\right)}{\sqrt{1+\delta_{1,0}}} \mathbf{G}_{\mathbf{l}}^{(\mathrm{c})}+\sum_{\mathbf{l} \neq \mathbf{0}}^{\prime} \sqrt{2} \sin \left(\mathbf{K}_{\mathbf{l}} \cdot \mathbf{r}\right) \mathbf{G}_{\mathbf{l}}^{(\mathrm{s})}
\end{aligned}
$$

To obtain amplitudes $\mathbf{G}_{1}^{(\mathrm{c})}$ and $\mathbf{G}_{1}^{(\mathrm{s})}$, we multiply $\mathbf{g}(\mathbf{r})$ with some cosine or sine function, and use the orthogonality between these basis functions when integrated over a unit cell $\Omega_{\mathrm{uc}}$ with a 
volume $V_{\mathrm{uc}}=\mathbf{R}_{1} \cdot\left(\mathbf{R}_{2} \times \mathbf{R}_{3}\right)$ to extract them $\left(\mathbf{l} \neq \mathbf{0}\right.$ for $\left.\mathbf{G}_{1}^{(\mathrm{s})}\right)$ :

$$
\begin{aligned}
& \mathbf{G}_{\mathbf{l}}^{(\mathrm{c})}=\sum_{\mathrm{l}^{\prime}}{ }^{\prime} \mathcal{N}_{1, \mathrm{I}^{\prime}}^{(\mathrm{cc})}[\omega] \mathbf{U}_{\mathbf{1}^{\prime}}^{(\mathrm{cc})}+\sum_{\mathrm{I}^{\prime} \neq \mathbf{0}}{ }^{\prime} \mathcal{N}_{\mathrm{l}, \mathrm{I}^{\prime}}^{(\mathrm{cs})}[\omega] \mathbf{U}_{\mathrm{l}^{\prime}}^{(\mathrm{s})} \\
& \mathbf{G}_{\mathbf{l}}^{(\mathrm{s})}=\sum_{\mathrm{l}^{\prime}}{ }^{\prime} \mathcal{N}_{1, \mathrm{I}^{\prime}}^{(\mathrm{sc})}[\omega] \mathbf{U}_{\mathrm{l}^{\prime}}^{(\mathrm{c})}+\sum_{\mathbf{l}^{\prime} \neq \mathbf{0}}{ }^{\prime} \mathcal{N}_{\mathrm{l}, \mathrm{I}^{\prime}}^{(\mathrm{ss})}[\omega] \mathbf{U}_{\mathrm{l}^{\prime}}^{(\mathrm{s})}
\end{aligned}
$$

where the four matrices $\mathcal{N}_{1, l^{\prime}}^{(\mathrm{cc})}[\omega], \mathcal{N}_{1, I^{\prime}}^{(\mathrm{cs})}[\omega], \mathcal{N}_{1,1^{\prime}}^{(\mathrm{sc})}[\omega]$, and $\mathcal{N}_{1,1^{\prime}}^{(s)}[\omega]$ are $\left(\mathbf{l}, \mathbf{l}^{\prime} \neq \mathbf{0}\right.$ if associated with sine functions $)$

$$
\begin{aligned}
& \mathcal{N}_{1, \mathrm{I}^{\prime}}^{(\mathrm{cc})}[\omega]=\frac{2}{V_{\mathrm{uc}}} \int_{\Omega_{\mathrm{uc}}} \mathrm{d} \mathbf{r} \mathbf{N}[\mathbf{r}, \omega] \frac{\cos \left(\mathbf{K}_{\mathbf{l}} \cdot \mathbf{r}\right)}{\sqrt{1+\delta_{1,0}}} \frac{\cos \left(\mathbf{K}_{\mathbf{1}^{\prime}} \cdot \mathbf{r}\right)}{\sqrt{1+\delta_{\mathbf{l}^{\prime}, 0}}} \\
& \mathcal{N}_{1, \mathrm{I}^{\prime}}^{(\mathrm{cs})}[\omega]=\frac{2}{V_{\mathrm{uc}}} \int_{\Omega_{\mathrm{uc}}} \mathrm{d} \mathbf{r} \mathbf{N}[\mathbf{r}, \omega] \frac{\cos \left(\mathbf{K}_{\mathrm{l}} \cdot \mathbf{r}\right)}{\sqrt{1+\delta_{\mathrm{l}, 0}}} \sin \left(\mathbf{K}_{\mathrm{l}^{\prime}} \cdot \mathbf{r}\right) \\
& \mathcal{N}_{1,1^{\prime}}^{(\mathrm{sc})}[\omega]=\frac{2}{V_{\mathrm{uc}}} \int_{\Omega_{\mathrm{uc}}} \mathrm{d} \mathbf{r} \mathbf{N}[\mathbf{r}, \omega] \sin \left(\mathbf{K}_{\mathbf{l}} \cdot \mathbf{r}\right) \frac{\cos \left(\mathbf{K}_{\mathrm{l}^{\prime}} \cdot \mathbf{r}\right)}{\sqrt{1+\delta_{\mathbf{1}^{\prime}, \mathbf{0}}}} \\
& \mathcal{N}_{\mathrm{l}, \mathrm{I}^{\prime}}^{(\mathrm{ss})}[\omega]=\frac{2}{V_{\mathrm{uc}}} \int_{\Omega_{\mathrm{uc}}} \mathrm{d} \mathbf{r} \mathbf{N}[\mathbf{r}, \omega] \sin \left(\mathbf{K}_{\mathbf{l}} \cdot \mathbf{r}\right) \sin \left(\mathbf{K}_{\mathrm{l}^{\prime}} \cdot \mathbf{r}\right) .
\end{aligned}
$$

From (51a) and (51b), the submatrix $\mathcal{N}_{1, \mathrm{I}^{\prime}}[\omega]$ of $\mathcal{N}[\omega]$ is expressed as

$$
\begin{aligned}
& \mathcal{N}_{1, l^{\prime}}[\omega]=\left(\begin{array}{cc}
\mathcal{N}_{1, I^{\prime}}^{(\mathrm{cc})}[\omega] & \mathcal{N}_{1, \mathrm{I}^{\prime}}^{(\mathrm{cs})}[\omega] \\
\mathcal{N}_{1, \mathrm{l}^{\prime}}^{(\mathrm{sc})}[\omega] & \mathcal{N}_{1, \mathrm{I}^{\prime}}^{(\mathrm{ss})}[\omega]
\end{array}\right), \quad \mathbf{1}, \mathrm{l}^{\prime} \neq \mathbf{0} \\
& \mathcal{N}_{1,0}[\omega]=\left(\begin{array}{c}
\mathcal{N}_{1,0}^{(\mathrm{cc})}[\omega] \\
\mathcal{N}_{1,0}^{(\mathrm{sc})}[\omega]
\end{array}\right), \quad \mathbf{1} \neq \mathbf{0} \\
& \mathcal{N}_{0,1}[\omega]=\left(\begin{array}{ll}
\mathcal{N}_{0,1}^{(\mathrm{cc})}[\omega] & \mathcal{N}_{\mathbf{0}, 1}^{(\mathrm{cs})}[\omega]
\end{array}\right), \quad \mathbf{1} \neq \mathbf{0} \\
& \mathcal{N}_{0,0}[\omega]=\mathcal{N}_{0,0}^{(\mathrm{cc})}[\omega] \text {. }
\end{aligned}
$$

Due to the symmetry $\mathbf{N}[\mathbf{r}, \omega]=\mathbf{N}^{\mathrm{T}}[\mathbf{r}, \omega]$, inspections of (52) reveal

$\mathcal{N}_{\mathbf{l}^{\prime}, 1}^{(\mathrm{cc}) \mathrm{T}}[\omega]=\mathcal{N}_{\mathbf{1}^{\prime}, 1}^{(\mathrm{cc})}[\omega]=\mathcal{N}_{1, \mathrm{I}^{\prime}}^{(\mathrm{cc}) \mathrm{T}}[\omega]=\mathcal{N}_{1, \mathrm{I}^{\prime}}^{(\mathrm{cc})}[\omega]$

$\mathcal{N}_{\mathrm{l}^{\prime}, 1}^{(\mathrm{cs}) \mathrm{T}}[\omega]=\mathcal{N}_{\mathrm{l}^{\prime}, 1}^{(\mathrm{cs})}[\omega]=\mathcal{N}_{1,1^{\prime}}^{(\mathrm{sc}) \mathrm{T}}[\omega]=\mathcal{N}_{1,1^{\prime}}^{(\mathrm{sc})}[\omega], \quad \mathbf{l} \neq \mathbf{0}$

$\mathcal{N}_{\mathrm{l}^{\prime}, 1}^{(\mathrm{ss}) \mathrm{T}}[\omega]=\mathcal{N}_{\mathrm{l}^{\prime}, 1}^{(\mathrm{ss})}[\omega]=\mathcal{N}_{1,1^{\prime}}^{(\mathrm{ss}) \mathrm{T}}[\omega]=\mathcal{N}_{1, \mathrm{I}^{\prime}}^{(\mathrm{ss})}[\omega], \quad \mathbf{l}, \mathbf{1}^{\prime} \neq \mathbf{0}$.

With (54), we then show $\mathcal{N}_{1^{\prime}, 1}^{\mathrm{T}}[\omega]=\mathcal{N}_{1,1^{\prime}}[\omega]$. Only submatrices with $\mathbf{l}^{\prime}, \mathbf{l} \neq \mathbf{0}$ are examined, and those with indices related to $\mathbf{0}$ can be carried out in a similar fashion:

$$
\begin{aligned}
\mathcal{N}_{\mathrm{l}^{\prime}, 1}^{\mathrm{T}}[\omega] & =\left(\begin{array}{ll}
\mathcal{N}_{\mathrm{l}^{\prime}, 1}^{(\mathrm{cc} T \mathrm{~T}}[\omega] & \mathcal{N}_{\mathrm{l}^{\prime}, 1}^{(\mathrm{sc}) \mathrm{T}}[\omega] \\
\mathcal{N}_{1^{\prime}, 1}^{(\mathrm{cs}) \mathrm{T}}[\omega] & \mathcal{N}_{1^{\prime}, 1}^{(\mathrm{ss}) \mathrm{T}}[\omega]
\end{array}\right) \\
& =\left(\begin{array}{ll}
\mathcal{N}_{1,1^{\prime}}^{(\mathrm{cc})}[\omega] & \mathcal{N}_{1,1^{\prime}}^{(\mathrm{cs})}[\omega] \\
\mathcal{N}_{1,1^{\prime}}^{(\mathrm{sc})}[\omega] & \mathcal{N}_{1,1^{\prime}}^{(\mathrm{ss})}[\omega]
\end{array}\right)=\mathcal{N}_{1, l^{\prime}}[\omega] .
\end{aligned}
$$

(55) indicates that the representation $\mathcal{N}[\omega]$ is symmetric.

\section{APPENDIX B}

\section{BAND Diagram OF BILAYER CHIRAL PHOTONIC CRYSTAL}

To calculate the band diagrams of the 1-D chiral PhC in Fig. 1, we use the free-space modes in each layer to expand the fields within it and apply the phase boundary condition with a phase factor $\exp \left(i k_{x} a\right)$ across one bilayer period. In this way, we can obtain the characteristic equation for nontrivial solutions and calculate the dispersion relations from it.

With a finite $k_{y}$ but null $k_{z}$, there are four free-space modes (labeled by $m=1-4)$ in layer $w(w=1,2)$. Here, we only consider their field components that are transverse to the $x$ direction because it is the transverse components of the total fields that are continuous across the slab boundary:

$$
\begin{gathered}
\left(\begin{array}{c}
E_{y, w, m}(x, y) \\
E_{z, w, m}(x, y) \\
\eta_{0} H_{y, w, m}(x, y) \\
\eta_{0} H_{z, w, m}(x, y)
\end{array}\right)=e^{i k_{y} y}\left[e^{i \beta_{x, w, m} x} \mathbf{u}_{w, m}\right] \\
\beta_{x, w, 1(2)}= \pm \beta_{x, w}^{(+)}= \pm \sqrt{\beta_{w}^{(+) 2}-k_{y}^{2}} \\
\beta_{x, w, 3(4)}= \pm \beta_{x, w}^{(-)}= \pm \sqrt{\beta_{w}^{(-) 2}-k_{y}^{2}} \\
\beta_{w}^{( \pm)}=k_{0}\left(\sqrt{\epsilon_{\mathrm{r}, w} \mu_{\mathrm{r}, w}} \pm \chi_{\mathrm{r}, w}\right)
\end{gathered}
$$

where $\beta_{x, w, m}$ is the propagation constant of mode $m$ in layer $w$ along the $x$ direction; $\beta_{w}^{( \pm)}$are the two chiral propagation constants; $\beta_{x, w}^{( \pm)}$are the corresponding $x$ components; and $\mathbf{u}_{w, m}$ are the composite amplitudes transverse to the $x$ direction. We use $\mathbf{u}_{w, m}(m=1-4)$ to construct a matrix $\mathbf{U}_{w}$ as

$$
\begin{aligned}
\mathbf{U}_{w} & =\left(\mathbf{u}_{w, 1}, \mathbf{u}_{w, 2}, \mathbf{u}_{w, 3}, \mathbf{u}_{w, 4}\right) \\
& =\left(\begin{array}{cccc}
-\frac{i \beta_{x, m}^{(+)}}{\beta^{(+)}} & \frac{i \beta_{x, m}^{(+)}}{\beta^{(+)}} & \frac{i \beta_{x, m}^{(-)}}{\beta^{(-)}} & -\frac{i \beta_{x, m}^{(-)}}{\beta^{(-)}} \\
1 & 1 & 1 & 1 \\
-\frac{\beta_{x, m}^{(+)}}{\eta_{\mathrm{r}, w} \beta^{(+)}} & \frac{\beta_{x, m}^{(+)}}{\eta_{\mathrm{r}, w} \beta^{(+)}} & -\frac{\beta_{x, m}^{(-)}}{\eta_{\mathrm{r}, w} \beta^{(-)}} & \frac{\beta_{x, m}^{(-)}}{\eta_{\mathrm{r}, w} \beta^{(-)}} \\
-\frac{i}{\eta_{\mathrm{r}, w}} & -\frac{i}{\eta_{\mathrm{r}, w}} & \frac{i}{\eta_{\mathrm{r}, w}} & \frac{i}{\eta_{\mathrm{r}, w}}
\end{array}\right)
\end{aligned}
$$

where $\eta_{\mathrm{r}, w}=\sqrt{\mu_{\mathrm{r}, w} / \epsilon_{\mathrm{r}, w}}$ is the relative impedance in layer $w$.

Dropping the exponential factor $\exp \left(i k_{y} y\right)$, we first expand the transverse composite fields at the rightmost boundary of layer 1 as $\sum_{m=1}^{4} \mathbf{u}_{1, m} A_{1, m}=\mathbf{U}_{1} \mathbf{A}_{1}$, where $A_{1, m}$ is the expansion coefficient of mode $m$ and also the $m$ th element of vector $\mathbf{A}_{1}$. We then convert the vector of expansion coefficients $\mathbf{A}_{1}$ into that $\mathbf{A}_{2}$ of layer 2 by demanding the continuity of the transverse composite amplitude through the boundary. By propagating $\mathbf{A}_{2}$ through layer 2 in the $+x$ direction, converting it back to the expansion vector of layer 1 in the next period, propagating it again to the rightmost boundary, and applying the phase boundary condition with phase factor $\exp \left(i k_{x} a\right)$, we 
obtain the following matrix equation:

$$
\left[\mathbf{U}_{1} \mathbf{Q}_{1}\left(a_{1}\right) \mathbf{U}_{1}^{-1} \mathbf{U}_{2} \mathbf{Q}_{2}\left(a_{2}\right) \mathbf{U}_{2}^{-1}\right] \mathbf{U}_{1} \mathbf{A}_{1}=e^{i k_{x} a} \mathbf{U}_{1} \mathbf{A}_{1}
$$

where $\mathbf{Q}_{w}\left(a_{w}\right)$ is the propagation matrix with matrix elements $\left[\mathbf{Q}_{w}\left(a_{w}\right)\right]_{m m^{\prime}}=\delta_{m m^{\prime}} \exp \left(i \beta_{x, w, m} a_{w}\right)$ in layer $w$. To have nontrivial solutions for the matrix equation in (58), we demand the following determinant to vanish:

$$
\operatorname{det}\left|\mathbf{U}_{1} \mathbf{Q}_{1}\left(a_{1}\right) \mathbf{U}_{1}^{-1} \mathbf{U}_{2} \mathbf{Q}_{2}\left(a_{2}\right) \mathbf{U}_{2}^{-1}-e^{i k_{x} a} \mathbf{I}_{4}\right|=0
$$

where $\mathbf{I}_{4}$ is the 4-by-4 identity matrix. (59) is then numerically solved to obtain the band diagrams in Fig. 2.

\section{REFERENCES}

[1] R. E. Collin, Field Theory of Guided Waves, 2nd ed. New York, NY, USA: IEEE Press, 1991.

[2] R. Carminati, M. Nieto-Vesperinas, and J.-J. Greffet, "Reciprocity of evanescent electromagnetic waves," J. Opt. Soc. Amer. A, vol. 15, no. 3, pp. 706-712, Mar. 1998.

[3] R. Carminati, J. J. Sáenz, J.-J. Greffet, and M. Nieto-Vesperinas, "Reciprocity, unitarity, and time-reversal symmetry of the s matrix of fields containing evanescent components," Phys. Rev. A, vol. 62, p. 012712, Jun. 2000.

[4] R. J. Potton, "Reciprocity in optics," Rep. Progr. Phys., vol. 67, no. 5, pp. 717-754, Apr. 2004.

[5] S. L. Chuang, "A coupled mode formulation by reciprocity and a variational principle," J. Lightw. Technol., vol. 5, no. 1, pp. 5-15, Jan. 1987.

[6] S. L. Chuang, "A coupled-mode theory for multiwaveguide systems satisfying the reciprocity theorem and power conservation," J. Lightw. Technol., vol. 5, no. 1, pp. 174-183, Jan. 1987.

[7] L. Tsang and S. L. Chuang, "Improved coupled-mode theory for reciprocal anisotropic waveguides," J. Lightw. Technol., vol. 6, no. 2, pp. 304-311, Feb. 1988.

[8] D. Michaelis, U. Peschel, C. Wächter, and A. Bräuer, "Reciprocity theorem and perturbation theory for photonic crystal waveguides," Phys. Rev. E, vol. 68, p. 065601, Dec. 2003.

[9] L. C. Botten, T. P. White, A. A. Asatryan, T. N. Langtry, C. Martijn de Sterke, and R. C. McPhedran, "Bloch mode scattering matrix methods for modeling extended photonic crystal structures. I. theory," Phys. Rev. E, vol. 70, p. 056606, Nov. 2004.

[10] A. T. Villeneuve and R. F. Harrington, "Reciprocity relationships for gyrotropic media," IRE Trans. Microw. Theory Tech., vol. 6, no. 3, pp. 308310, Jul. 1958.

[11] A. T. Villeneuve, "Orthogonality relationships for waveguides and cavities with inhomogeneous anisotropic media," IRE Trans. Microw. Theory Tech., vol. 7, no. 4, pp. 441-446, Oct. 1959.

[12] F. Olyslager, "Properties of and generalized full-wave transmission line models for hybrid (bi)(an)isotropic waveguides," IEEE Trans. Microw. Theory Tech., vol. 44, no. 11, pp. 2064-2075, Nov. 1996.

[13] V. Dmitriev, "Comments on "properties of and generalized full-wave transmission line models for hybrid (bi)(an)isotropic waveguides', "IEEE Trans. Microw. Theory Tech., vol. 47, no. 5, pp. 655-657, May 1999

[14] F. Olyslager, "Author's reply," IEEE Trans. Microw. Theory Tech., vol. 47, no. 5, pp. 658-659, May 1999.

[15] F. Olyslager, Electromagnetic Waveguides and Transmission Lines. Oxford, U.K.: Oxford Univ. Press, 1999.
[16] D. Pissoort and F. Olyslager, "Study of eigenmodes in periodic waveguides using the lorentz reciprocity theorem," IEEE Trans. Microw. Theory Tech., vol. 52, no. 2, pp. 542-553, Feb. 2004.

[17] A. D. Yaghjian, "Bidirectionality of reciprocal, lossy or lossless, uniform or periodic waveguides," IEEE Microw. Wireless Compon. Lett., vol. 17, no. 7, pp. 480-482, Jul. 2007.

[18] F. Olyslager and H. Rogier, "Bidirectionality in the waveguide sturmliouville problem (invited paper)," in Proc. EMTS 2007-Int. URSI Commiss. B-Electromagn. Theory Symp., Jul. 2007.

[19] H. A. Kramers, "General theory of paramagnetic rotation in crystals," Proc. Amsterdam Acad., vol. 33, pp. 959-972, 1930.

[20] E. Wigner, "Über die operation der zeitumkehr in der quantenmechanik," Nachr. Akad. Ges. Wiss. Göttingen, vol. 31, pp. 546-559, 1932.

[21] O. Madelung, Introduction to Solid-State Theory, 3rd ed. Berlin, Germany: Springer-Verlag, 1996.

[22] P. J. Chiang and S. W. Chang, "Frequency-domain formulation of photonic crystals using sources and gain," Opt. Exp., vol. 21, no. 2, pp. 1972-1985, Jan. 2013.

[23] S. W. Chang, "Full frequency-domain approach to reciprocal microlasers and nanolasers-perspective from lorentz reciprocity," Opt. Exp., vol. 19, no. 22 , pp. $21116-21134$, Oct. 2011

[24] J. R. Carson, "A generalization of reciprocal theorem," Bell Syst. Techn. J., vol. 3, no. 3, pp. 393-399, Jul. 1924.

[25] J. R. Carson, "Reciprocal theorems in radio communication," Proc. IRE., vol. 17 , no. 6, pp. 952-956, Jun. 1929.

[26] J. A. Kong, Electromagnetic Wave Theory, 7th ed. Cambridge, MA, USA: EMW Publishing, 2008.

[27] S. H. Friedberg, A. J. Insel, and L. E. Spence, Linear Algebra, 2nd ed. Englewood Cliffs, NJ, USA: Prentice-Hall, 1989.

Shu-Wei Chang (M'09) received the B.S. degree in electrical engineering from the National Taiwan University, Taipei, Taiwan, in 1999, and the M.S. and Ph.D. degrees from the Department of Electrical and Computer Engineering, University of Illinois at Urbana-Champaign, Urbana, IL, USA, in 2003 and 2006 , respectively.

From 2008 to 2010, he was a Postdoctorate Associate with the Department of Electrical and Computer Engineering, University of Illinois at UrbanaChampaign. Since 2010, he has been an Assistant Research Fellow with the Research Center for Applied Sciences, Academia Sinica, Taipei. In 2011, he joined the faculties of the Department of Photonics, National Chiao-Tung University, Hsinchu, Taiwan, as an Adjunct Assistant Professor. His research interest include fundamental and applied physics of semiconductor photonics including tunneling-injection quantum-dot-quantum-well coupled system, slow and fast light in semiconductor nanostructures, spin relaxation in strained [110] and [111] semiconductor quantum wells, group-IV direct-bandgap semiconductor lasers, active and passive plasmonic devices, semiconductor nanolasers, applications of metamaterials, both chiral and nonchiral, to semiconductor active devices, and computational schemes for both reciprocal and nonreciprocal cavities.

Dr. Chang is a member of the Optical Society of America. He received the John Bardeen Memorial Graduate Award from the Department of Electrical and Computer Engineering, University of Illinois at Urbana-Champaign, in 2006. 